\title{
8-RANKS OF CLASS GROUPS OF IMAGINARY QUADRATIC NUMBER FIELDS AND THEIR DENSITIES
}

\author{
Hwanyup Jung and Qin Yue
}

\begin{abstract}
For imaginary quadratic number fields $F=\mathbb{Q}\left(\sqrt{\varepsilon p_{1} \cdots p_{t-1}}\right)$, where $\varepsilon \in\{-1,-2\}$ and distinct primes $p_{i} \equiv 1 \bmod 4$, we give conditions of 8-ranks of class groups $C(F)$ of $F$ equal to 1 or 2 provided that 4-ranks of $C(F)$ are at most equal to 2. Especially for $F=\mathbb{Q}\left(\sqrt{\varepsilon p_{1} p_{2}}\right)$, we compute densities of 8-ranks of $C(F)$ equal to 1 or 2 in all such imaginary quadratic fields $F$. The results are stated in terms of congruence relations of $p_{i}$ modulo $2^{n}$, the quartic residue symbol $\left(\frac{p_{1}}{p_{2}}\right)_{4}$ and binary quadratic forms such as $p_{2}^{h_{+}\left(2 p_{1}\right) / 4}=x^{2}-2 p_{1} y^{2}$, where $h_{+}\left(2 p_{1}\right)$ is the narrow class number of $\mathbb{Q}\left(\sqrt{2 p_{1}}\right)$. The results are also very useful for numerical computations.
\end{abstract}

\section{Introduction}

It is a classical topic to study the structure of 2-primary subgroups of the narrow class groups $C_{+}(F)$ for quadratic number fields $F([1,2,3,9,12,13,14])$. Gerth gave a method to compute their densities $([4,5,6,15,16])$. By genus theory, we have known 2-rank of $C_{+}(F)$; by Rédei's matrix, we have got 4-rank of $C_{+}(F)$ clearly. In this paper, we always assume that $F=\mathbb{Q}\left(\sqrt{\varepsilon p_{1} \cdots p_{t-1}}\right)$, where $\varepsilon \in\{-1,-2\}$, are imaginary quadratic number fields with distinct primes $p_{i} \equiv 1 \bmod 4$. We will mainly obtain conditions for 8-ranks of class groups $C(F)$ equal to 1 or 2 provided that 4-ranks of $C(F)$ are at most equal to 2 . Especially for $F=\mathbb{Q}\left(\sqrt{\varepsilon p_{1} p_{2}}\right)$, we compute densities of 8-ranks of $C(F)$ equal to 1 or 2 in all such fields.

In $\S 2$, we describe some well-known facts. We support the degree 4 extension $N_{+}$over $K=\mathbb{Q}\left(\sqrt{2 p_{1}}\right)$ with prime $p_{1} \equiv 1 \bmod 8$, in which all finite primes of $K$ are unramified. We set up relations between the Galois group $\operatorname{Gal}\left(N_{+} / K\right)$ and the narrow class group $C_{+}(K)$ of $K$. We represent general Legendre symbols

Received July 13, 2010; Revised December 13, 2010.

2010 Mathematics Subject Classification. 11R29, 11R45.

Key words and phrases. class group, unramified extension, quartic residue, density.

The first author was supported by Basic Science Research Program through the National Research Foundation of Korea(NRF) funded by the Ministry of Education, Science and Technology(2011-0005138).

The second author was partly supported by NNSF of China(No. 10771100, No. 10971250). 
by binary quadratic forms $q^{h_{+}(2 p) / 4}=x^{2}-2 p y^{2}$ and $\pm p_{2}^{h_{+}\left(2 p_{1}\right) / 4}=2 x^{2}-p_{1} y^{2}$ over $\mathbb{Z}$, where $h_{+}\left(2 p_{1}\right)$ is the narrow class number of $K$. Meanwhile, we give some quartic reciprocity laws.

In $\S 3$, we investigate 8-ranks of class groups $C(F)$ for imaginary quadratic fields $F=\mathbb{Q}\left(\sqrt{\varepsilon p_{1} \cdots p_{t-1}}\right)$, where $\varepsilon \in\{-1,-2\}$ and distinct primes $p_{i} \equiv$ $1 \bmod 4$. We give the necessary and sufficient conditions for 8-ranks of $C(F)$ equal to 1 or 2 provided that 4-ranks of $C(F)$ are at most equal to 2 . Their results are expressed by congruence relations of $p_{i}$ modulo $2^{n}$, general Legendre symbols and quartic residue symbols $\left(\frac{p_{1}}{p_{2}}\right)_{4},\left(\frac{2 p_{1}}{p_{2}}\right)_{4}$ (see [10]). These results are very useful for numerical calculations.

In $\S 4$, especially for $F=\mathbb{Q}\left(\sqrt{\varepsilon p_{1} p_{2}}\right)$, we compute densities for 8-ranks of $C(F)$ equal to 1 or 2 in such quadratic number fields (Theorem 4.1).

We use the following notation:

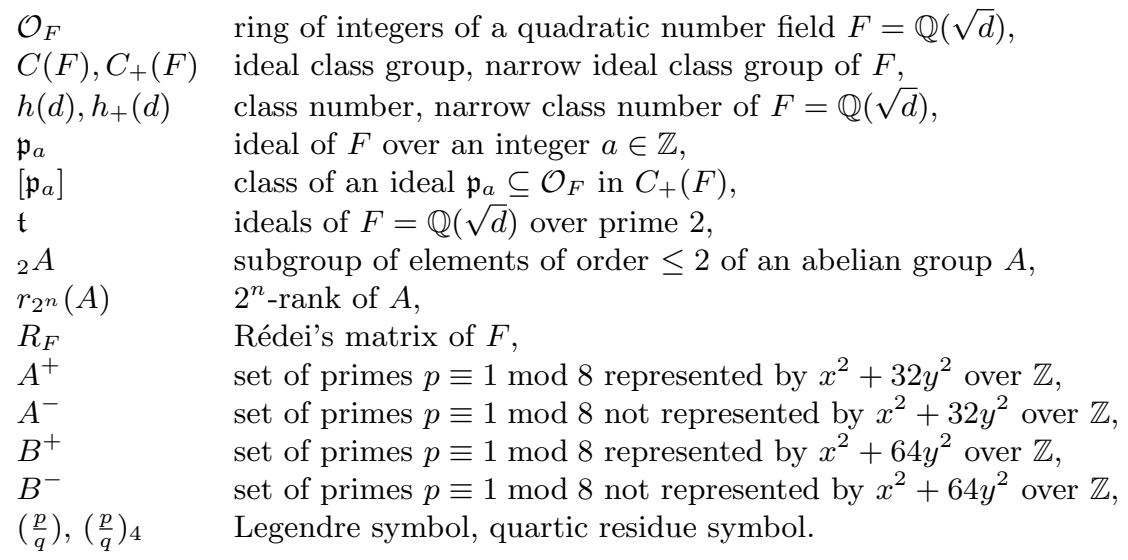

\section{Preliminaries}

First, for a prime $p_{1} \equiv 1 \bmod 8$, we find the cyclic extension $N_{+}$of degree 4 over $K=\mathbb{Q}\left(\sqrt{2 p_{1}}\right)$, in which no finite prime of $K$ ramifies. In terms of norm from $L=\mathbb{Q}(\sqrt{2})$ over $\mathbb{Q}, p_{1}=u_{1}^{2}-2 w_{1}^{2}$ with $u_{1}, w_{1} \in \mathbb{Z}$ and, without loss of generality, we shall always assume that

$$
\pi_{1}=u_{1}+w_{1} \sqrt{2} \in L \text { with } u_{1} \equiv 1 \bmod 4, w_{1} \equiv 0 \bmod 4,
$$

which is called a primary element in $L$. In fact, $w_{1}$ is even and we can multiply $u_{1}+w_{1} \sqrt{2}$ by the element $(1+\sqrt{2})^{2}=3+2 \sqrt{2}$ of norm 1 , if necessary. By genus theory, 2-primary subgroup of the narrow class group $C_{+}(K)$ of $K$ is a cyclic and $4 \mid h_{+}\left(2 p_{1}\right)$. Let $N_{+}=\mathbb{Q}\left(\sqrt{2}, \sqrt{p_{1}}, \sqrt{\pi_{1}}\right)$. It is clear that $N_{+}$is a normal extension of degree 8 over $\mathbb{Q}$. Consider the tower of relative quadratic 
extensions:

$$
\begin{gathered}
N_{+}=\mathbb{Q}\left(\sqrt{2}, \sqrt{p_{1}}, \sqrt{\pi_{1}}\right) \\
K_{1}=\mathbb{Q}\left(\sqrt{2}, \sqrt{p_{1}}\right) \\
K=\mathbb{Q}\left(\sqrt{2 p_{1}}\right) \\
\mid
\end{gathered}
$$

Let $\mathfrak{t}$ and $\mathfrak{p}_{1}$ be the prime ideals of $K$ over 2 and $p_{1}$, respectively. We can verify that $\mathfrak{t}$ and $\mathfrak{p}_{1}$ are unramified in $N_{+}$, so all finite primes of $K$ are unramified in $N_{+}$(in details, see [3]). Moreover, if $p_{1} \in A^{+}$, then $u_{1} \in \mathbb{N}$ by [1], so $N_{+}$is the unramified cyclic extension of degree 4 over $K$.

Let $p_{2} \equiv 1 \bmod 8$ be a prime. Then $p_{2}=u_{2}^{2}-2 w_{2}^{2}$ with $u_{2}, w_{2} \in \mathbb{Z}$, and

$$
\pi_{2}=u_{2}+w_{2} \sqrt{2} \in L \text { with } u_{2} \equiv 1 \bmod 4, w_{2} \equiv 0 \bmod 4 .
$$

Suppose $\left(\frac{p_{1}}{p_{2}}\right)=1$, so $p_{2}$ splits completely in $K_{1}$. Let $\mathfrak{p}_{2}^{\prime}=\pi_{2} \mathcal{O}_{L}=\left(\pi_{2}\right)$ be a prime ideal of $L$ over $p_{2}$ and $\mathcal{P}_{2}$ be a prime ideal of $K_{1}$ over $\mathfrak{p}_{2}^{\prime}$, i.e., $\mathfrak{p}_{2}^{\prime} \mid p_{2}$ and $\mathcal{P}_{2} \mid \mathfrak{p}_{2}^{\prime}$. Then $\mathcal{O}_{K_{1}} / \mathcal{P}_{2} \cong \mathcal{O}_{L} / \mathfrak{p}_{2}^{\prime} \cong \mathbb{Z} /\left(p_{2}\right)$. Hence the general Legendre symbol $([8$, p. 196])

$$
\left(\frac{\pi_{1}}{\mathcal{P}_{2}}\right)=\left(\frac{\pi_{1}}{\mathfrak{p}_{2}^{\prime}}\right)
$$

which is denoted by $\left(\frac{\pi_{1}}{\pi_{2}}\right)$. In fact,

$$
\left(\frac{\pi_{1}}{\pi_{2}}\right)=1 \Leftrightarrow x^{2} \equiv \pi_{1} \bmod \pi_{2} \mathcal{O}_{L} \text { has a solution in } \mathcal{O}_{L} \text {. }
$$

Since $\mathcal{O}_{L} / \mathfrak{p}_{2}^{\prime} \cong \mathbb{Z} /\left(p_{2}\right)$ and $\left(\frac{p_{1}}{p_{2}}\right)=1,\left(\frac{\pi_{1}}{\pi_{2}}\right)=\left(\frac{\bar{\pi}_{1}}{\pi_{2}}\right)$, where $\bar{\pi}_{1}=u_{1}-w_{1} \sqrt{2}$ is the conjugate element of $\pi_{1}$. Hence $p_{2}$ splits completely in $L_{1}=\mathbb{Q}\left(\sqrt{2}, \sqrt{\pi_{1}}\right)$ if and only if $\left(\frac{\pi_{1}}{\pi_{2}}\right)=1$. By the reciprocity law ([8, Theorem 165]), we have $\left(\frac{\pi_{1}}{\pi_{2}}\right)=\left(\frac{\pi_{2}}{\pi_{1}}\right)$. Therefore $p_{2}$ splits completely in $N_{+}$if and only if $\left(\frac{\pi_{1}}{\pi_{2}}\right)=1$. We have proved:

Lemma 2.1. Let $p_{1} \equiv p_{2} \equiv 1 \bmod 8$ be primes with $\left(\frac{p_{1}}{p_{2}}\right)=1$ and $\pi_{1}, \pi_{2}$ be defined as above. Then

(i) $p_{2}$ splits completely in $N_{+}$if and only if $\left(\frac{\pi_{1}}{\pi_{2}}\right)=1$.

(ii) $p_{2}$ splits completely in $K_{1}$ but does not in $N_{+}$if and only if $\left(\frac{\pi_{1}}{\pi_{2}}\right)=-1$.

In the following, we use the binary quadratic form to describe the value of $\left(\frac{\pi_{1}}{\pi_{2}}\right)$. Let $H_{+}(K)$ be the narrow Hilbert class field of $K$, which is the maximal abelian extension over $K$ in which no finite prime of $K$ ramifies. Then $\operatorname{Gal}\left(H_{+}(K) / K\right) \cong C_{+}(K)$ and $K \subset K_{1} \subset N_{+} \subset H_{+}(K)$. Especially, if $p_{1} \in A^{+}$, then $N_{+} \subset H(K)$, which is the Hilbert class field of $K$. By restriction there is an epimorphism: $C_{+}(K) \rightarrow \operatorname{Gal}\left(N_{+} / K\right)$, where $\operatorname{Gal}\left(N_{+} / K\right)$ is cyclic of order 4 . Hence

$$
C_{+}(K) / C_{+}(K)^{4} \cong \operatorname{Gal}\left(N_{+} / K\right)
$$


and analogously

$$
C_{+}(K) / C_{+}(K)^{2} \cong \operatorname{Gal}\left(K_{1} / K\right) .
$$

Let $\mathfrak{p}$ be a prime ideal of $\mathcal{O}_{K}$. We have that $\mathfrak{p}$ splits completely in $N_{+} \Leftrightarrow$ the Artin symbol $\left(\frac{N_{+} / K}{\mathfrak{p}}\right)=1 \in \operatorname{Gal}\left(N_{+} / K\right) \Leftrightarrow[\mathfrak{p}] \in C_{+}(K)^{4}$ (see [11, p. 104]). Let $\mathfrak{p}_{2}$ be a prime ideal of $\mathcal{O}_{K}$ over $p_{2}$. Then we conclude that $\mathfrak{p}_{2}$ splits completely in $N_{+} \Leftrightarrow\left(\frac{\pi_{1}}{\pi_{2}}\right)=1 \Leftrightarrow\left[\mathfrak{p}_{2}\right] \in C_{+}(K)^{4} \Leftrightarrow\left[\mathfrak{p}_{2}\right]^{h_{+}}\left(2 p_{1}\right) / 4=1 \Leftrightarrow$ $p_{2}^{h_{+}\left(2 p_{1}\right) / 4}=x^{2}-2 p_{1} y^{2}$ for some $x, y \in \mathbb{Z}$.

Let $\mathfrak{t}$ and $\mathfrak{p}_{1}$ be prime ideals of $\mathcal{O}_{K}$ over 2 and $p_{1}$, respectively. By genus theory, $[\mathfrak{t}],\left[\mathfrak{p}_{1}\right]$ and $\left[\mathfrak{t p}_{1}\right]$ are of order at most 2 and only one of them is the unit in $C_{+}(K)$. Suppose $[\mathfrak{t}]$ is of order 2 . Then we have that $\mathfrak{p}_{2}$ splits completely in $K_{1}$ but does not in $N_{+} \Leftrightarrow\left(\frac{\pi_{1}}{\pi_{2}}\right)=-1 \Leftrightarrow\left[\mathfrak{p}_{2}\right] \in C_{+}(K)^{2}$ and $\left[\mathfrak{p}_{2}\right] \notin C_{+}(K)^{4}$ $\Leftrightarrow[\mathfrak{t}]\left[\mathfrak{p}_{2}\right]^{h_{+}\left(2 p_{1}\right) / 4}=1 \in C_{+}(K) \Leftrightarrow p_{2}^{h_{+}\left(2 p_{1}\right) / 4}=2 x^{2}-p_{2} y^{2}$ for some $x, y \in \mathbb{Z}$. Suppose $[\mathfrak{t}]=1$ and $\left[\mathfrak{p}_{1}\right]$ is of order 2 . Then, similarly, we have that $\left(\frac{\pi_{1}}{\pi_{2}}\right)=-1$ $\Leftrightarrow\left[\mathfrak{p}_{1}\right]\left[\mathfrak{p}_{2}\right]^{h_{+}\left(2 p_{1}\right) / 4}=1 \in C_{+}(K) \Leftrightarrow p_{2}^{h_{+}\left(2 p_{1}\right) / 4}=p_{1} x^{2}-2 y^{2}$ for some $x, y \in \mathbb{Z}$. Hence we have proved:

Lemma 2.2. Let $p_{1} \equiv p_{2} \equiv 1 \bmod 8$ be primes with $\left(\frac{p_{1}}{p_{2}}\right)=1$. Then

(i) $\left(\frac{\pi_{1}}{\pi_{2}}\right)=1$ if and only if $p_{2}^{h_{+}\left(2 p_{1}\right) / 4}=x^{2}-2 p_{1} y^{2}$ for some $x, y \in \mathbb{Z}$.

(ii) $\left(\frac{\pi_{1}}{\pi_{2}}\right)=-1$ if and only if $\pm p_{2}^{h_{+}\left(2 p_{1}\right) / 4}=2 x^{2}-p_{1} y^{2}$ for some $x, y \in \mathbb{Z}$.

Moreover, for $p_{2}=u_{2}^{2}-2 w_{2}^{2} \equiv 1 \bmod 8$, we have that $\left(\frac{w_{2}}{p_{2}}\right)=1=\left(\frac{w_{2}}{\pi_{2}}\right)$. Since $p_{2}=2\left(u_{2}+w_{2}\right)^{2}-\left(u_{2}+2 w_{2}\right)^{2}$ and $u_{2}+w_{2} \equiv w_{2}(1-\sqrt{2}) \bmod \pi_{2} \mathcal{O}_{L}$, by [1], we conclude that

$$
\begin{gathered}
p_{2} \in A^{+} \Leftrightarrow u_{2}>0, u_{2}+w_{2}>0 \Leftrightarrow\left(\frac{u_{2}+w_{2}}{p_{2}}\right)=\left(\frac{1-\sqrt{2}}{\pi_{2}}\right)=1 ; \\
\left(\frac{u_{2}}{p_{2}}\right)=1 \Leftrightarrow\left(\frac{2}{p_{2}}\right)_{4}=1 \Leftrightarrow p_{2} \in B^{+} .
\end{gathered}
$$

Now we describe some results about quartic reciprocity law. Let $p_{1} \equiv p_{2} \equiv$ $1 \bmod 4$ be distinct primes. Then $p_{1}=a_{1}^{2}+b_{1}^{2}, p_{2}=a_{2}^{2}+b_{2}^{2}, b_{1} \equiv b_{2} \equiv 0 \bmod 2$, over $\mathbb{Z}$ in terms of norm from $L_{1}=\mathbb{Q}(i)$, where $i=\sqrt{-1}$. We shall always assume that

$$
\lambda_{1}=a_{1}+i b_{1}, \lambda_{2}=a_{2}+i b_{2} \text { with } a_{1}+b_{1} \equiv a_{2}+b_{2} \equiv 1 \bmod 4,
$$

which are called primary elements in $L_{1}$.

For any $\alpha \in \mathbb{Z}[i]$ with $\lambda_{1} \nmid \alpha$, there exists a unique integer $j(0 \leq j \leq 3)$ such that

$$
\alpha^{\frac{N\left(\lambda_{1}\right)-1}{4}} \equiv i^{j} \bmod \lambda_{1} \mathcal{O}_{L_{1}} \text {. }
$$

We will define by $\left(\frac{\alpha}{\lambda_{1}}\right)_{4}=i^{j}$ the quartic residue symbol of $\alpha$ modulo $\lambda_{1}$. There is a fact that $\left(\frac{p_{2}}{\lambda_{1}}\right)_{4}=1$ if and only if $x^{4} \equiv p_{2} \bmod p_{1}$ has a solution with $x \in \mathbb{Z}$, 
which is denoted by $\left(\frac{p_{2}}{p_{1}}\right)_{4}=1$. There is the law of quartic reciprocity (see $[10$, p.123]):

$$
\left(\frac{\lambda_{1}}{\lambda_{2}}\right)_{4}=\left(\frac{\lambda_{2}}{\lambda_{1}}\right)_{4}(-1)^{\frac{\left(p_{1}-1\right)\left(p_{2}-1\right)}{16}}
$$

Lemma 2.3. Let $p_{1} \equiv p_{2} \equiv 1 \bmod 4$ be distinct primes, $p_{1}=a_{1}^{2}+b_{1}^{2}, p_{2}=$ $a_{2}^{2}+b_{2}^{2}$, and $\lambda_{1}=a_{1}+i b_{1}, \lambda_{2}=a_{2}+i b_{2}$ be primary elements as above.

(i) If $\left(\frac{p_{1}}{p_{2}}\right)=1$, then $\left(\frac{p_{1}}{p_{2}}\right)_{4}\left(\frac{p_{2}}{p_{1}}\right)_{4}=\left(\frac{\lambda_{2}}{\lambda_{1}}\right)$.

(ii) Suppose $p_{1} \equiv p_{2} \equiv 5 \bmod 8$ and $\left(\frac{p_{1}}{p_{2}}\right)=-1$. Then

$$
\left(\frac{2 p_{1}}{p_{2}}\right)_{4}\left(\frac{2 p_{2}}{p_{1}}\right)_{4}=i^{\frac{p_{1}+p_{2}-2}{4}}\left(\frac{\lambda_{2}}{\lambda_{1}}\right)
$$

where we take $a_{1}+b_{1} \equiv a_{2}+b_{2} \equiv 1 \bmod 8$.

Proof. (i) Let $p_{1}=\lambda_{1} \bar{\lambda}_{1}$ and $p_{2}=\lambda_{2} \bar{\lambda}_{2}$, where $\bar{\lambda}_{1}$ and $\bar{\lambda}_{2}$ are the conjugate elements of $\lambda_{1}$ and $\lambda_{2}$, respectively. By the quartic reciprocity law, we have that

$$
\begin{aligned}
\left(\frac{p_{1}}{p_{2}}\right)_{4}\left(\frac{p_{2}}{p_{1}}\right)_{4}=\left(\frac{p_{1}}{\lambda_{2}}\right)_{4}\left(\frac{p_{2}}{\lambda_{1}}\right)_{4} & =\left(\frac{\lambda_{1}}{\lambda_{2}}\right)_{4}\left(\frac{\bar{\lambda}_{1}}{\lambda_{2}}\right)_{4}\left(\frac{\lambda_{2}}{\lambda_{1}}\right)_{4}\left(\frac{\bar{\lambda}_{2}}{\lambda_{1}}\right)_{4} \\
& =\left(\frac{\lambda_{2}}{\lambda_{1}}\right)_{4}^{2}\left(\frac{\lambda_{2}}{\bar{\lambda}_{1}}\right)_{4}\left(\frac{\bar{\lambda}_{2}}{\lambda_{1}}\right)_{4}=\left(\frac{\lambda_{2}}{\lambda_{1}}\right)
\end{aligned}
$$

where $\left(\frac{\lambda_{2}}{\bar{\lambda}_{1}}\right)_{4}\left(\frac{\bar{\lambda}_{2}}{\lambda_{1}}\right)_{4}=1$.

(ii) Similarly, we have that

$$
\begin{aligned}
\left(\frac{2 p_{1}}{p_{2}}\right)_{4}\left(\frac{2 p_{2}}{p_{1}}\right)_{4} & =\left(\frac{2 p_{1}}{\lambda_{2}}\right)_{4}\left(\frac{2 p_{2}}{\lambda_{1}}\right)_{4} \\
& =\left(\frac{2}{\lambda_{1} \lambda_{2}}\right)_{4}\left(\frac{p_{1}}{\lambda_{2}}\right)_{4}\left(\frac{p_{2}}{\lambda_{1}}\right)_{4} \\
& =\left(\frac{2}{\lambda_{1} \lambda_{2}}\right)_{4}\left(\frac{\lambda_{2}}{\lambda_{1}}\right) .
\end{aligned}
$$

Since $p_{1} \equiv 5 \bmod 8$ and $2 p_{1}=\left(a_{1}+b_{1}\right)^{2}+\left(a_{1}-b_{1}\right)^{2}$, we assume that $a_{1}+b_{1} \equiv$ $1 \bmod 8$ and $a_{1}-b_{1} \equiv 5 \bmod 8$. Similarly, we may assume that $a_{2}+b_{2} \equiv$ $1 \bmod 8$ and $a_{2}-b_{2} \equiv 5 \bmod 8$. By [10, p. 136, Ex.37], we have $\left(\frac{1+i}{\lambda_{1}}\right)_{4}=$ $i^{\left(a_{1}-b_{1}-b_{1}^{2}-1\right) / 4}$. Since $2=i^{3}(1+i)^{2}$ and $\left(\frac{i}{\lambda_{1}}\right)_{4}=i^{(p-1) / 4}$, we have

$$
\left(\frac{2}{\lambda_{1}}\right)_{4}\left(\frac{2}{\lambda_{2}}\right)_{4}=i^{\frac{3\left(p_{1}-1+p_{2}-1\right)}{4}+\frac{a_{1}-b_{1}-b_{1}^{2}-1+a_{2}-b_{2}-b_{2}^{2}-1}{2}}=i^{\frac{p_{1}+p_{2}-2}{4}} \text {. }
$$

In fact, since $a_{1}+b_{1} \equiv a_{2}+b_{2} \equiv 1 \bmod 8, a_{1}-b_{1}-b_{1}^{2}-1=a_{1}+b_{1}-\left(b_{1}+1\right)^{2} \equiv$ $0 \bmod 8$ and $a_{2}-b_{2}-b_{2}^{2}-1=a_{2}+b_{2}-\left(b_{2}+1\right)^{2} \equiv 0 \bmod 8$. 


\section{Elements of order 8}

Let $F=\mathbb{Q}(\sqrt{D})$ be a quadratic field and $D$ be the discriminant of $F$. The prime discriminant is either $p^{*}=(-1)^{(p-1) / 2} p$ if $p$ is an odd prime or $p^{*}=-4,8,-8$ if $p=2$. Then $D$ has the unique decomposition $D=p_{1}^{*} \cdots p_{t}^{*}$ into a product of prime discriminants and $p_{t}=2$ if $2 \mid D$. By genus theory, $r_{2}\left(C_{+}(F)\right)=t-1$.

We will denote by $\left(\frac{n}{p}\right)$ the Legendre symbol if $p$ is an odd prime and by $\left(\frac{n}{2}\right)$ the Kronecker symbol. If $\left(\frac{n}{p}\right)=(-1)^{a}$ with $a \in \mathbb{F}_{2}$, we shall write $\left(\frac{n}{p}\right)^{\prime}=a$. Then the Rédei matrix $R_{F}=\left(a_{i j}\right)$ of $F$ is the $t \times t$ matrix with $a_{i j} \in \mathbb{F}_{2}$ given by

$$
a_{i j}=\left\{\begin{array}{ll}
\left(\frac{p_{i}^{*}}{p_{j}}\right)^{\prime} & \text { if } i \neq j, \\
\left(\frac{D / p_{i}^{*}}{p_{i}}\right)^{\prime} & \text { if } i=j,
\end{array} \quad \text { for } 1 \leq i, j \leq t .\right.
$$

Note that the sum of all rows of $R_{F}$ is equal to 0 . Let $R_{F}^{\prime}$ be the $(t-1) \times t$ matrix obtained from $R_{F}$ by deleting the $t$-th row. Then $\operatorname{rank} R_{F}^{\prime}=\operatorname{rank} R_{F}$, where the rank is always meant to the rank over $\mathbb{F}_{2}$.

Let $D(F)$ be the set of all positive square-free divisors $q$ of the discriminant $D$. Then $D(F)$ is an elementary abelian 2-group with multiplication $q_{1} \cdot q_{2}=$ $q_{1} q_{2} /\left(q_{1}, q_{2}\right)^{2}$, where $\left(q_{1}, q_{2}\right)$ is the greatest common divisor of $q_{1}, q_{2}$. For $q \in$ $D(F)$, we define $X_{q}=\left(x_{1}, \ldots, x_{t}\right)^{T} \in \mathbb{F}_{2}^{t}$ by

$$
x_{i}=\left\{\begin{array}{ll}
1 & \text { if } p_{i} \mid q, \\
0 & \text { if } p_{i} \nmid q,
\end{array} \quad \text { for } 1 \leq i \leq t .\right.
$$

Then we have that $R_{F}^{\prime} X_{q}=0 \Leftrightarrow\left(\frac{q}{p}\right)=1$ for every odd prime $p \mid(D / q)$ and $\left(\frac{-D / q}{p}\right)=1$ for every odd prime $p \mid q \Leftrightarrow x^{2}-D y^{2}=q z^{2}$ is solvable over $\mathbb{Z} \Leftrightarrow$ $q \in D(F) \cap N_{F / \mathbb{Q}}\left(F^{*}\right)$. Hence,

$$
\theta: D(F) \cap N_{F / \mathbb{Q}}\left(F^{*}\right) \rightarrow\left\{X_{q}: R_{F}^{\prime} X_{q}=0\right\}, q \mapsto X_{q},
$$

is an isomorphism. By genus theory, $\alpha: D(F) \cap N_{F / \mathbb{Q}}\left(F^{*}\right) \rightarrow{ }_{2} C(F) \cap C(F)^{2}$ is surjective and $|\operatorname{Ker}(\alpha)|=2$. We have the Rédei's criterion:

$$
r_{4}\left(C_{+}(F)\right)=r_{2}\left(D(F) \cap N_{F / \mathbb{Q}}\left(F^{*}\right)\right)-1=t-1-\operatorname{rank} R_{F} .
$$

We know the method of Rédei's matrix to determine the solutions of the Diophantine equations $q z^{2}=x^{2}-D y^{2}$ over $\mathbb{Z}$. For convenience, if it has a nontrivial solution over $\mathbb{Z}$, then it will be called solvable.

Let $F=\mathbb{Q}(\sqrt{-d})$ be an imaginary quadratic field with $d=p_{1} \cdots p_{t-1}$ and distinct primes $p_{i} \equiv 1 \bmod 4$. Then the narrow class group $C_{+}(F)$ is just the class group $C(F)$ and $r_{2}(C(F))=t-1$ by genus theory. The Rédei's matrix 
of $F$ is

$$
R_{F}=\left(\begin{array}{cccc}
\left(\frac{D / p_{1}^{*}}{p_{1}}\right)^{\prime} & \cdots & \left(\frac{p_{t-1}}{p_{1}}\right)^{\prime} & \left(\frac{p_{t}}{p_{1}}\right)^{\prime} \\
\vdots & & \vdots & \vdots \\
\left(\frac{p_{1}}{p_{t-1}}\right)^{\prime} & \cdots & \left(\frac{D / p_{t-1}^{*}}{p_{t-1}}\right)^{\prime} & \left(\frac{p_{t}}{p_{t}-1}\right)^{\prime} \\
0 & \cdots & 0 & \left(\frac{p_{t}}{p_{1} \cdots p_{t-1}}\right)^{\prime}
\end{array}\right)=\left(\begin{array}{cc}
M & \alpha \\
0 & \left(\frac{p_{t}}{p_{1} \cdots p_{t-1}}\right)^{\prime}
\end{array}\right),
$$

where $p_{t}=2$ and $M$ is equal to the $(t-1) \times(t-1)$ Rédei's matrix $R_{E}$ of the real quadratic field $E=\mathbb{Q}(\sqrt{d})$.

Proposition 3.1. Let $F=\mathbb{Q}(\sqrt{-d})$ be an imaginary quadratic field with $d=$ $p_{1} \cdots p_{t-1}$ and distinct primes $p_{i} \equiv 1 \bmod 4(t \geq 3)$. Let $E=\mathbb{Q}(\sqrt{d})$ be a real quadratic field. Then

(i) $r_{4}(C(F))=0$ if and only if $d \equiv 5 \bmod 8$ and $r_{4}\left(C_{+}(E)\right)=0$.

(ii) $r_{4}(C(F))=r(1 \leq r \leq t-1)$ if and only if either $r_{4}\left(C_{+}(E)\right)=r-1$ and $q \equiv 1 \bmod 8$ for each $q \in D(E)$ or $r_{4}\left(C_{+}(E)\right)=r$ and there is some $q \in D(E)$ such that $q \equiv 5 \bmod 8$.

Proof. (i) Since $p_{i} \equiv 1 \bmod 4$ for $1 \leq i \leq t-1, R_{E}$ is a symmetric matrix and $\operatorname{rank} R_{E} \leq t-2$. By Rédei's criterion, $r_{4}(C(F))=0 \Leftrightarrow \operatorname{rank} R_{F}=t-1 \Leftrightarrow$ $\operatorname{rank} R_{E}=t-2$ and $\left(\frac{2}{p_{1} \cdots p_{t-1}}\right)=-1 \Leftrightarrow r_{4}\left(C_{+}(E)\right)=0$ and $d \equiv 5 \bmod 8$.

(ii) Suppose $r_{4}(C(F))=r$, so rank $R_{F}=t-1-r$. Note that the sum of all row vectors of $R_{F}$ is equal to zero vector. We have that $\operatorname{rank} R_{F}=t-1-r$ if and only if either rank $R_{E}=t-1-r$ and the vector $\alpha$ is linearly represented by column vectors of $R_{E}$ in (3.1) or $\operatorname{rank} R_{E}=t-1-r-1$ and $\alpha$ is not linearly represented by column vectors of $R_{E}$. We only need to prove that $\alpha$ is linearly represented by column vectors of $R_{E}$ if and only if $q \equiv 1 \bmod 8$ for each $q \in D(E)$.

If $\alpha$ is linearly represented by column vectors of $R_{E}$ and $q=p_{1} \cdots p_{s} \in D(E)$ $(s \leq t-1)$, then $R_{E} X_{q}=0$, where $X_{q}$ is a vector corresponding with $q \in D(E)$. Hence, since $R_{E}$ is a symmetric matrix, the addition with the first $s$ columns (rows) of $R_{E}$ is equal to zero vector, so $\left(\frac{2}{p_{1} \cdots p_{s}}\right)=1$, i.e., $q=p_{1} \cdots p_{s} \equiv$ $1 \bmod 8$.

Conversely, since $d=p_{1} \cdots p_{t-1} \in D(E), d \equiv 1 \bmod 8$ and $\left(\frac{2}{p_{1} \cdots p_{t-1}}\right)=1$, we need prove $\operatorname{rank}\left(R_{E}, \alpha\right)=\operatorname{rank} R_{E}$. Without loss of generality, we assume that the first $k=t-1-r$ rows $\beta_{1}, \ldots, \beta_{k}$ of $R_{E}$ is a maximal subset of linearly independent of all rows of $R_{E}$. If, for a row $\beta_{i}(k<i \leq t-1)$ of $R_{E}$, we have $\beta_{1}+\cdots+\beta_{k}+\beta_{i}=0$, then $q=p_{1} \cdots p_{k} p_{i} \in D(E)$ and $q \equiv 1 \bmod 8$. Let

$$
M^{\prime}=\left(\begin{array}{cc}
\beta_{1} & \left(\frac{2}{p_{1}}\right)^{\prime} \\
\vdots & \vdots \\
\beta_{k} & \left(\frac{2}{p_{k}}\right)^{\prime} \\
\beta_{i} & \left(\frac{2}{p_{i}}\right)^{\prime}
\end{array}\right)
$$


Then $\left(\frac{2}{p_{1}}\right)^{\prime}+\cdots+\left(\frac{2}{p_{k}}\right)^{\prime}+\left(\frac{2}{p_{i}}\right)^{\prime}=0$ and $\operatorname{rank} M^{\prime}=k$, so the last row of $M^{\prime}$ is linearly represented by the first $k$ rows of $M^{\prime}$. Hence $\operatorname{rank}\left(R_{E}, \alpha\right)=\operatorname{rank} R_{E}$ and $\alpha$ is linearly represented by column vectors of $R_{E}$.

Write $D^{*}(F)=D(F) \cap N_{F / \mathbb{Q}}\left(F^{*}\right)$ for simplicity.

Remark 3.2. By the process of proving Proposition 3.1, we have that

(i) $r_{4}(C(F))=r_{4}\left(C_{+}(E)\right)$ if and only if $D^{*}(F)=D^{*}(E)$;

(ii) $r_{4}(C(F))=r_{4}\left(C_{+}(E)\right)+1$ if and only if there is some $q \mid p_{1} \cdots p_{t-1}$ such that $2 q z^{2}=x^{2}+p_{1} \cdots p_{t-1} y^{2}$ is solvable if and only if $2 q \in D^{*}(F)$.

By Proposition 3.1, we have that $r_{4}(C(F))=1$ if and only if one of the following conditions holds:

(1) $\operatorname{rank} R_{F}=\operatorname{rank} R_{E}+1=t-2$ and $D^{*}(F)=D^{*}(E)=\left\{1, q_{1}, q_{2}, d\right\}$, where at least one of $q_{1}=p_{1} \cdots p_{r}$ and $q_{2}=p_{r+1} \cdots p_{t-1}$ is congruent to 5 modulo $8(1 \leq r<t-1)$;

(2) $\operatorname{rank} R_{F}=\operatorname{rank} R_{E}=t-2$ and $p_{1} \cdots p_{t-1} \equiv 1 \bmod 8$, so $D^{*}(F)=$ $\left\{1,2 q_{1}, 2 q_{2}, d\right\}$, where $q_{1}=p_{1} \cdots p_{r}$ and $q_{2}=p_{r+1} \cdots p_{t-1}(0 \leq r<t-1$ and $q_{1}=1$ if $\left.r=0\right)$.

Theorem 3.3. Let $F=\mathbb{Q}(\sqrt{-d})$, where $d=p_{1} \cdots p_{t-1}$ with distinct primes $p_{i} \equiv 1 \bmod 4$, be an imaginary quadratic field and $r_{4}(C(F))=1$.

(i) Suppose $D^{*}(F)=\left\{1, q_{1}, q_{2}, d\right\}$, where $q_{1}=p_{1} \cdots p_{r} \equiv 1 \bmod 8$ and $q_{2}=p_{r+1} \cdots p_{t-1} \equiv 5 \bmod 8$. Then $r_{8}(C(F))=1$ if and only if $\left(\frac{q_{2}}{q_{1}}\right)_{4}=$ 1.

(ii) Suppose $D^{*}(F)=\left\{1, q_{1}, q_{2}, d\right\}$, where $q_{1}=p_{1} \cdots p_{r} \equiv 5 \bmod 8$ and $q_{2}=p_{r+1} \cdots p_{t-1} \equiv 5 \bmod 8$. Then $r_{8}(C(F))=1$ if and only if $\left(\frac{q_{1}}{q_{2}}\right)_{4}\left(\frac{q_{2}}{q_{1}}\right)_{4}=-1$.

(iii) Suppose $D^{*}(F)=\left\{1,2 q_{1}, 2 q_{2}, d\right\}$, where $q_{1}=p_{1} \cdots p_{r} \equiv 5 \bmod 8$ and $q_{2}=p_{r+1} \cdots p_{t-1} \equiv 5 \bmod 8$. Then $r_{8}(C(F))=1$ if and only if either $d \equiv 9 \bmod 16$ and $\left(\frac{2 q_{1}}{q_{2}}\right)_{4}\left(\frac{2 q_{2}}{q_{1}}\right)_{4}=-1$ or either $d \equiv 1 \bmod 16$ and $\left(\frac{2 q_{1}}{q_{2}}\right)_{4}\left(\frac{2 q_{2}}{q_{1}}\right)_{4}=1$.

(iv) Suppose $D^{*}(F)=\left\{1,2 q_{1}, 2 q_{2}, d\right\}$, where $q_{1}=p_{1} \cdots p_{r} \equiv 1 \bmod 8$ and $q_{2}=p_{r+1} \cdots p_{t-1} \equiv 1 \bmod 8$. Then $r_{8}(C(F))=1$ if and only if either $d \equiv 1 \bmod 16$ and $\left(\frac{2 q_{1}}{q_{2}}\right)_{4}\left(\frac{2 q_{2}}{q_{1}}\right)_{4}=-1$ or either $d \equiv 9 \bmod 16$ and $\left(\frac{2 q_{1}}{q_{2}}\right)_{4}\left(\frac{2 q_{2}}{q_{1}}\right)_{4}=1$.

Proof. (i) Suppose rank $R_{F}=t-2, D^{*}(F)=\left\{1, q_{1}, q_{2}, d\right\}$ and $q_{1}=p_{1} \cdots p_{r} \equiv$ $1 \bmod 8, q_{2}=p_{r+1} \cdots p_{t-1} \equiv 5 \bmod 8$. Then the sum of the first $r$ row vectors of $R_{F}$ is equal to zero vector. Let $\mathfrak{q}_{1}^{2}=q_{1} \mathcal{O}_{F}$. Then $1 \neq\left[\mathfrak{q}_{1}\right] \in{ }_{2} C(F) \cap C(F)^{2}$. By Rédei's criterion, $z^{2}=q_{1} x^{2}+q_{2} y^{2}$ has a relatively prime solution $(x, y, z)=$ $(a, b, c)$ over $\mathbb{N}$, so $\left[\mathfrak{q}_{1}\right]=[\mathfrak{c}]^{2} \in C(F)^{2}$, where $\mathfrak{c}$ is an ideal of $\mathcal{O}_{F}$ over $c$. Since $c^{2}=q_{1} a^{2}+q_{2} b^{2}$ and $q_{1} \equiv 1 \bmod 8$, we have that the Jacobi symbols $\left(\frac{b}{q_{1}}\right)=1$ and $\left(\frac{c}{q_{1}}\right)=\left(\frac{q_{2}}{q_{1}}\right)_{4}$, where $\left(\frac{q_{2}}{q_{1}}\right)_{4}=\left(\frac{q_{2}}{p_{1}}\right)_{4} \cdots\left(\frac{q_{2}}{p_{r}}\right)_{4}$. We conclude that $r_{8}(C(F))=1 \Leftrightarrow\left[\mathfrak{q}_{1}\right] \in C(F)^{4} \Leftrightarrow[\mathfrak{c}][\mathfrak{m}] \in C(F)^{2}$, where $\mathfrak{m}$ is an ambiguous 
ideal of $F$ over $m \mid 2 d \Leftrightarrow m c z^{2}=x^{2}+d y^{2}$ is solvable over $\mathbb{Z} \Leftrightarrow$ the following system of equations is solvable over $\mathbb{F}_{2}$

$$
R_{F}^{\prime} X=\left(\begin{array}{c}
\left(\frac{c}{p_{1}}\right)^{\prime} \\
\vdots \\
\left(\frac{c}{p_{t-1}}\right)^{\prime}
\end{array}\right)
$$

$\Leftrightarrow\left(\frac{c}{q_{1}}\right)=\left(\frac{c}{p_{1} \cdots p_{r}}\right)=1=\left(\frac{c}{q_{1}}\right)_{4}$ (since $\left.\operatorname{rank} R_{F}^{\prime}=t-2\right)$.

(ii) Suppose $\operatorname{rank} R_{F}=t-2, D^{*}(F)=\left\{1, q_{1}, q_{2}, d\right\}$ and $q_{1}=p_{1} \cdots p_{r} \equiv$ $5 \bmod 8, q_{2}=p_{r+1} \cdots p_{t-1} \equiv 5 \bmod 8$. Then the sum of the first $t-1$ row vectors of $R_{F}$ is equal to zero and the sum of the first $r$ row vectors of $M$ is also equal to zero. Let $z^{2}=q_{1} x^{2}+q_{2} y^{2}$ have a non-trivial solution $(x, y, z)=$ $(a, b, c)$ over $\mathbb{N}$. Then, by Rédei's criterion, $r_{4}(C(F))=1$ and $1 \neq\left[\mathfrak{q}_{1}\right]=$ $[\mathfrak{c}]^{2} \in{ }_{2} C(F) \cap C(F)^{2}$, where $\mathfrak{q}_{1}^{2}=q_{1} \mathcal{O}_{F}$ and $\mathfrak{c}$ is an ideal of $F$ over $c$. Since $q_{1} \equiv q_{2} \equiv 5 \bmod 8$, without loss of generality, $c^{2}=q_{1} a^{2}+4 q_{2} b^{\prime 2}$, where $b=2 b^{\prime}$ and $a \equiv b^{\prime} \equiv 1 \bmod 2$. Hence the Jacobi symbol $\left(\frac{a}{q_{2}}\right)=1=\left(\frac{b^{\prime}}{q_{1}}\right)=-\left(\frac{b}{q_{1}}\right)$. Since $c^{2}=q_{1} a^{2}+q_{2} b^{2}$, we have that $\left(\frac{c}{q_{1}}\right)=\left(\frac{q_{2}}{q_{1}}\right)_{4}\left(\frac{b}{q_{1}}\right)$ and $\left(\frac{c}{q_{2}}\right)=\left(\frac{q_{1}}{q_{2}}\right)_{4}\left(\frac{a}{q_{2}}\right)$. Similarly, we conclude that

$$
r_{8}(C(F))=1 \Leftrightarrow\left[\mathfrak{q}_{1}\right] \in C(F)^{4} \Leftrightarrow\left(\frac{c}{q_{1}}\right)=\left(\frac{c}{q_{2}}\right) \Leftrightarrow\left(\frac{q_{1}}{q_{2}}\right)_{4}\left(\frac{q_{2}}{q_{1}}\right)_{4}=-1 .
$$

(iii) Suppose $\operatorname{rank} R_{F}=t-2$ and $D^{*}(F)=\left\{1,2 q_{1}, 2 q_{2}, d\right\}$, where $q_{1}=$ $p_{1} \cdots p_{r} \equiv 5 \bmod 8$ and $q_{2}=p_{r+1} \cdots p_{t-1} \equiv 5 \bmod 8$. Then the sum of the first $t-1$ row vectors of $R_{F}$ is equal to zero vector, i.e., $\left(\frac{2}{p_{1} \cdots p_{t-1}}\right)=1$. Let $2 z^{2}=q_{1} x^{2}+q_{2} y^{2}$ have a non-trivial solution $(x, y, z)=(a, b, c)$ over $\mathbb{N}$, where $a, b, c$ are all odd. Then $1 \neq\left[\mathfrak{t q}_{1}\right]=[\mathfrak{c}]^{2} \in C(F)^{2}$, where $\mathfrak{t}^{2}=2 \mathcal{O}_{F}, \mathfrak{q}_{1}^{2}=q_{1} \mathcal{O}_{F}$, and $\mathfrak{c}$ is an ideal of $F$ over $c$. Since $2 c^{2}=q_{1} a^{2}+q_{2} b^{2}$, we have that Jacobi symbols $\left(\frac{2 q_{2}}{a}\right)=\left(\frac{2 q_{1}}{b}\right)=1$ and

$$
\left(\frac{c}{q_{1}}\right)=\left(\frac{2 q_{2}}{q_{1}}\right)_{4}\left(\frac{b}{q_{1}}\right), \quad\left(\frac{c}{q_{2}}\right)=\left(\frac{2 q_{1}}{q_{2}}\right)_{4}\left(\frac{a}{q_{2}}\right) .
$$

Since $\left(q_{1} a\right)^{2}+d b^{2}=2 q_{1} c^{2} \equiv 10 \bmod 16$, we have that $d \equiv 9 \bmod 16 \Leftrightarrow 9 a^{2}+$ $9 b^{2} \equiv 10 \bmod 16 \Leftrightarrow a b \equiv \pm 3 \bmod 8 \Leftrightarrow\left(\frac{2}{a}\right)=-\left(\frac{2}{b}\right) \Leftrightarrow\left(\frac{a}{q_{2}}\right)=-\left(\frac{b}{q_{1}}\right) ;$ in other word, $d \equiv 1 \bmod 16 \Leftrightarrow\left(\frac{a}{q_{2}}\right)=\left(\frac{b}{q_{1}}\right)$. We conclude that $r_{8}(C(F))=1$ $\Leftrightarrow\left[\mathfrak{t a}_{1}\right] \in C(F)^{4} \Leftrightarrow\left(\frac{c}{d}\right)=1$, i.e., $\left(\frac{c}{q_{1}}\right)=\left(\frac{c}{q_{2}}\right) \Leftrightarrow$ either $d \equiv 9 \bmod 16$ with $\left(\frac{2 q_{2}}{q_{1}}\right)_{4}\left(\frac{2 q_{1}}{q_{2}}\right)_{4}=-1$ or $d \equiv 1 \bmod 16$ with

$$
\left(\frac{2 q_{2}}{q_{1}}\right)_{4}\left(\frac{2 q_{1}}{q_{2}}\right)_{4}=1
$$

(iv) It is clear from the process of proving (iii).

Let $F=\mathbb{Q}\left(\sqrt{-p_{1} p_{2}}\right)$ be an imaginary quadratic field with $p_{1} \equiv p_{2} \equiv 1 \mathrm{mod}$ 4. By Rédei's criterion, we have that $r_{4}(C(F))=1$ if and only if one of the following four conditions holds: 
(1) $p_{1} \equiv p_{2}+4 \equiv 1 \bmod 8$ and $\left(\frac{p_{1}}{p_{2}}\right)=1$;

(2) $p_{1} \equiv p_{2} \equiv 5 \bmod 8$ and $\left(\frac{p_{1}}{p_{2}}\right)=1$;

(3) $p_{1} \equiv p_{2} \equiv 5 \bmod 8$ and $\left(\frac{p_{1}}{p_{2}}\right)=-1$;

(4) $p_{1} \equiv p_{2} \equiv 1 \bmod 8$ and $\left(\frac{p_{1}}{p_{2}}\right)=-1$.

By Theorem 3.3 and Lemma 2.3, we have proved:

Corollary 3.4. Let $F=\mathbb{Q}\left(\sqrt{-p_{1} p_{2}}\right)$ be an imaginary quadratic field.

(i) Suppose $p_{1} \equiv 1 \bmod 8, p_{2} \equiv 5 \bmod 8$ and $\left(\frac{p_{1}}{p_{2}}\right)=1$. Then $r_{8}(C(F))=1$ if and only if $\left(\frac{p_{2}}{p_{1}}\right)_{4}=1$.

(ii) Suppose $p_{1} \equiv p_{2} \equiv 5 \bmod 8$ and $\left(\frac{p_{1}}{p_{2}}\right)=1$. Then $r_{8}(C(F))=1$ if and only if $\left(\frac{p_{2}}{p_{1}}\right)_{4}\left(\frac{p_{1}}{p_{2}}\right)_{4}=-1$ if and only if $\left(\frac{\lambda_{1}}{\lambda_{2}}\right)=1$, where $\lambda_{1}$ and $\lambda_{2}$ are defined as Lemma 2.3 .

(iii) Suppose $p_{1} \equiv p_{2} \equiv 5 \bmod 8$ and $\left(\frac{p_{1}}{p_{2}}\right)=-1$. Then $r_{8}(C(F))=1$ if and only if either $p_{1} p_{2} \equiv 9 \bmod 16$ and $\left(\frac{2 p_{1}}{p_{2}}\right)_{4}\left(\frac{2 p_{2}}{p_{1}}\right)_{4}=-1$ or $p_{1} p_{2} \equiv$ $1 \bmod 16$ and $\left(\frac{2 p_{1}}{p_{2}}\right)_{4}\left(\frac{2 p_{2}}{p_{1}}\right)_{4}=1$ if and only if $\left(\frac{\lambda_{1}}{\lambda_{2}}\right)=1$, where $\lambda_{1}$ and $\lambda_{2}$ are defined as Lemma 2.3.

(iv) Suppose $p_{1} \equiv p_{2} \equiv 1 \bmod 8$ and $\left(\frac{p_{1}}{p_{2}}\right)=-1$. Then $r_{8}(C(F))=1$ if and only if either $p_{1}, p_{2} \in A^{+}$or $p_{1}, p_{2} \in A^{-}$if and only if $\left(\frac{1-\sqrt{2}}{\pi_{1} \pi_{2}}\right)=1$, where $\pi_{1}$ and $\pi_{2}$ are defined as in $\S 2$.

Example 3.5. In Corollary 3.4, let $F=\mathbb{Q}\left(\sqrt{-p_{1} p_{2}}\right)$ with distinct primes $p_{1} \equiv p_{2} \equiv 1 \bmod 4$. Let $C(F)_{2}$ denote the 2-primary subgroup of $C(F)$.

(i) For $p_{1}=17$ and $p_{2}=13,\left(\frac{17}{13}\right)=1,3^{4}=13+17 \cdot 4,\left(\frac{13}{17}\right)_{4}=1$, so $r_{8}(C(F))=1$ by Theorem 3.3(i). In fact, $C(F)_{2} \cong \mathbb{Z} /(8) \oplus \mathbb{Z} /(2)$ by Pari-GP.

(ii) For $p_{1}=13$ and $p_{2}=29,\left(\frac{13}{29}\right)=1,13=3^{2}+2^{2}, 29=5^{2}+2^{2}$, $\left(\frac{13}{19}\right)_{4}\left(\frac{29}{13}\right)_{4}=-1$ by quartic reciprocity, so $r_{8}(C(F))=1$ by Theorem 3.3(ii). In fact, $C(F)_{2} \cong \mathbb{Z} /(8) \oplus \mathbb{Z} /(2)$ by Pari-GP.

(iii) For $p_{1}=13$ and $p_{2}=37,\left(\frac{37}{13}\right)=-1, p_{1} \cdot p_{2} \equiv 1 \bmod 16,2 \cdot 37=$ $4^{4}-14 \cdot 13,2 \cdot 17=11^{4}-395 \cdot 37,\left(\frac{2 \cdot 37}{13}\right)_{4}=\left(\frac{2 \cdot 13}{37}\right)_{4}=1$, so $r_{8}(C(F))=1$ by Theorem 3.3 (iii). In fact, $C(F)_{2} \cong \mathbb{Z} /(8) \oplus \mathbb{Z} /(2)$ by Pari-GP.

(iv) For $p_{1}=17$ and $p_{2}=73, p_{1}, p_{2} \in A^{-}, r_{8}(C(F))=1$ by Theorem 3.3(iv). In fact, $C(F)_{2} \cong \mathbb{Z} /(16) \oplus \mathbb{Z} /(2)$ by Pari-GP.

In Proposition 3.1, we know that $r_{4}(C(F))=2$ if and only if one of the following conditions holds:

(1) $\operatorname{rank} R_{F}=\operatorname{rank} R_{E}=t-3$ and $D(F)=\left(q_{1}\right) \times\left(2 q_{1}^{\prime}\right) \times(d)$, where $q_{1}=p_{1} \cdots p_{r} \equiv 1 \bmod 8(1 \leq r<t-1)$ and $q_{1}^{\prime} \mid d$.

(2) $\operatorname{rank} R_{F}=\operatorname{rank} R_{E}+1=t-3$ and $D(F)=D(E)=\left(q_{1}\right) \times\left(q_{2}\right) \times\left(q_{3}\right)$, where $q_{1}=p_{1} \cdots p_{r}, q_{2}=p_{r+1} \cdots p_{s}$ and $q_{3}=p_{s+1} \cdots p_{t-1}$.

Theorem 3.6. Let $F=\mathbb{Q}(\sqrt{-d})$, where $d=p_{1} \cdots p_{t-1}$ and distinct primes $p_{i} \equiv 1 \bmod 8$, be an imaginary quadratic field. Let $\operatorname{rank} R_{F}=t-3$ and $D(F)=\left(q_{1}\right) \times(2) \times(d)$, where $q_{1}=p_{1} \cdots p_{r}(1 \leq r<t-1)$. 
(i) Let $\mathfrak{q}_{1}^{2}=q_{1} \mathcal{O}_{F}$. Then $\left[\mathfrak{q}_{1}\right] \in C(F)^{4}$ if and only if $\left(\frac{q_{1}}{q_{2}}\right)_{4}=\left(\frac{q_{2}}{q_{1}}\right)_{4}=1$.

(ii) Let $p_{i}=u_{i}^{2}-2 w_{i}^{2} \equiv 1 \bmod 8$ and $\pi_{i}=u_{i}+w_{i} \sqrt{2}$ for $1 \leq i \leq t-1$. Let $\pi_{1}^{\prime}=\prod_{i=1}^{r} \pi_{i}=u_{1}^{\prime}+w_{1}^{\prime} \sqrt{2}, \pi_{2}^{\prime}=\prod_{i=r+1}^{t-1} \pi_{i}=u_{2}^{\prime}+w_{2}^{\prime} \sqrt{2}$ and $\mathfrak{t}^{2}=2 \mathcal{O}_{F}$. Then $[\mathfrak{t}] \in C(F)^{4}$ if and only if $\left(\frac{1-\sqrt{2}}{\pi_{1}^{\prime}}\right)=\left(\frac{1-\sqrt{2}}{\pi_{2}}\right)=\left(\frac{\pi_{1}^{\prime}}{\pi_{2}^{\prime}}\right)$ if and only if either both $p_{1}, \ldots, p_{r}$ and $p_{r+1}, \ldots, p_{t-1}$ belonging to $A^{-}$are two even numbers and $\left(\frac{\pi_{1}^{\prime}}{\pi_{2}^{\prime}}\right)=1$ or both $p_{1}, \ldots, p_{r}$ and $p_{r+1}, \ldots, p_{t-1}$ belonging to $A^{-}$are two odd numbers and $\left(\frac{\pi_{1}^{\prime}}{\pi_{2}^{\prime}}\right)=-1$. Moreover, $r_{8}(C(F))=2$ if and only if $\left[\mathfrak{q}_{1}\right],[\mathfrak{t}] \in C(F)^{4}$ if and only if $\left(\frac{q_{1}}{q_{2}}\right)_{4}=$ $\left(\frac{q_{2}}{q_{1}}\right)_{4}=1$ and $\left(\frac{1-\sqrt{2}}{\pi_{1}^{\prime}}\right)=\left(\frac{1-\sqrt{2}}{\pi_{2}^{\prime}}\right)=\left(\frac{\pi_{1}}{\pi_{2}^{\prime}}\right)$.

Proof. (i) Suppose rank $R_{F}=t-3$ and $D(F)=\left(q_{1}\right) \times(2) \times(d)$, where $q_{1}=$ $p_{1} \cdots p_{r}(1 \leq r<t-1)$. Then the two sums of both the first $r$ row vectors and the first $t-1$ row vectors of $R_{F}$ are equal to zero. Let $z^{2}=q_{1} x^{2}+q_{2} y^{2}$, $q_{2}=d / q_{1}$, have a non-trivial solution $(x, y, z)=(a, b, c)$ over $\mathbb{N}$. Then $1 \neq$ $\left[\mathfrak{q}_{1}\right]=[\mathfrak{c}]^{2} \in C(F)^{2}$, where $\mathfrak{q}_{1}^{2}=q_{1} \mathcal{O}_{F}$ and $\mathfrak{c}$ is an ideal of $F$ over $c$. Since $c^{2}=q_{1} a^{2}+q_{2} b^{2}$ and $q_{1} \equiv q_{2} \equiv 1 \bmod 8$, the Jacobi symbols $\left(\frac{a}{q_{2}}\right)=\left(\frac{b}{q_{1}}\right)=1$ and

$$
\left(\frac{c}{q_{1}}\right)=\left(\frac{q_{2}}{q_{1}}\right)_{4}, \quad\left(\frac{c}{q_{2}}\right)=\left(\frac{q_{1}}{q_{2}}\right)_{4} .
$$

We conclude that $\left[\mathfrak{q}_{1}\right] \in C(F)^{4} \Leftrightarrow[\mathfrak{c}][\mathfrak{m}] \in C(F)^{2}$, where $\mathfrak{m}$ is an ambiguous ideal of $F$ over $m \mid 2 d \Leftrightarrow m c z^{2}=x^{2}+d y^{2}$ is solvable over $\mathbb{Z} \Leftrightarrow$ the following system of equations is solvable over $\mathbb{F}_{2}$

$$
R_{F}^{\prime} X=\left(\begin{array}{c}
\left(\frac{c}{p_{1}}\right)^{\prime} \\
\vdots \\
\left(\frac{c}{p_{t-1}}\right)^{\prime}
\end{array}\right)
$$

$\Leftrightarrow\left(\frac{c}{q_{1}}\right)=\left(\frac{q_{2}}{q_{1}}\right)_{4}=1$ and $\left(\frac{c}{q_{2}}\right)=\left(\frac{q_{1}}{q_{2}}\right)_{4}=1$.

(ii) Since $q_{1} q_{2}=N_{L / \mathbb{Q}}\left(\pi_{1}^{\prime} \pi_{2}^{\prime}\right)=u^{2}-2 w^{2}=2(u+w)^{2}-(u+2 w)^{2}$, where $u=u_{1}^{\prime} u_{2}^{\prime}+2 w_{1}^{\prime} w_{2}^{\prime}$ and $w=u_{1}^{\prime} w_{2}^{\prime}+u_{2}^{\prime} w_{1}^{\prime}$, we have

$$
[\mathfrak{t}]=\left[\mathfrak{p}_{u+w}\right]^{2} \in C(F)^{2},
$$

where $\mathfrak{p}_{u+w}$ is an ideal of $F$ over $u+w$. For each $p_{i}(1 \leq i \leq r), \mathcal{O}_{L} /\left(\pi_{i}\right) \cong$ $\mathbb{Z} /\left(p_{i}\right)$ and $\left(\frac{u+w}{p_{i}}\right)=\left(\frac{u+w}{\pi_{i}}\right)$. On the other hand,

$$
\begin{aligned}
u+w & =u_{1}^{\prime} u_{2}^{\prime}+2 w_{1}^{\prime} w_{2}^{\prime}+u_{1}^{\prime} w_{2}^{\prime}+u_{2}^{\prime} w_{1}^{\prime} \\
& \equiv-w_{1}^{\prime} u_{2}^{\prime} \sqrt{2}+2 w_{1}^{\prime} w_{2}^{\prime}-w_{1}^{\prime} w_{2}^{\prime} \sqrt{2}+u_{2}^{\prime} w_{1}^{\prime} \\
& \equiv w_{1}^{\prime}(1-\sqrt{2})\left(u_{2}^{\prime}-w_{2}^{\prime} \sqrt{2}\right) \bmod \pi_{i},
\end{aligned}
$$

so

$$
\left(\frac{u+w}{p_{i}}\right)=\left(\frac{u+w}{\pi_{i}}\right)=\left(\frac{w_{1}^{\prime}}{\pi_{i}}\right)\left(\frac{1-\sqrt{2}}{\pi_{i}}\right)\left(\frac{\pi_{2}^{\prime}}{\pi_{i}}\right), 1 \leq i \leq r .
$$


Similarly, we get:

$$
\left(\frac{u+w}{p_{j}}\right)=\left(\frac{u+w}{\pi_{j}}\right)=\left(\frac{w_{2}^{\prime}}{\pi_{j}}\right)\left(\frac{1-\sqrt{2}}{\pi_{j}}\right)\left(\frac{\pi_{1}^{\prime}}{\pi_{j}}\right), r+1 \leq j \leq t-1 .
$$

Since $q_{1}=u_{1}^{\prime 2}-2 w_{1}^{\prime},\left(\frac{w_{1}^{\prime}}{q_{1}}\right)=\left(\frac{w_{1}^{\prime}}{\pi_{1}^{\prime}}\right)=1$, similarly, $\left(\frac{w_{2}^{\prime}}{q_{2}}\right)=\left(\frac{w_{2}^{\prime}}{\pi_{2}^{\prime}}\right)=1$. Note the fact that $p_{i} \in A^{+}$if and only if $\left(\frac{1-\sqrt{2}}{\pi_{i}}\right)=1$. By reciprocity law, we know that $\left(\frac{\pi_{1}^{\prime}}{\pi_{2}^{\prime}}\right)=\left(\frac{\pi_{2}^{\prime}}{\pi_{1}^{\prime}}\right)$. Since $\operatorname{rank} R_{F}=t-2$ and $p_{i} \equiv 1 \bmod 8$, we conclude that $[\mathfrak{t}] \in C(F)^{4} \Leftrightarrow$ the following system of equations is solvable over $\mathbb{F}_{2}$

$$
R_{F}^{\prime} X=\left(\begin{array}{c}
\left(\frac{u+w}{p_{1}}\right)^{\prime} \\
\vdots \\
\left(\frac{u+w}{p_{t-1}}\right)^{\prime}
\end{array}\right)
$$

$\Leftrightarrow\left(\frac{u+w}{q_{1}}\right)=1$ and $\left(\frac{u+w}{q_{2}}\right)=1 \Leftrightarrow$ either both $p_{1}, \ldots, p_{r}$ and $p_{r+1}, \ldots, p_{t-1}$ belonging to $A^{-}$are two even numbers and $\left(\frac{\pi_{1}^{\prime}}{\pi_{2}^{\prime}}\right)=1$ or both $p_{1}, \ldots, p_{r}$ and $p_{r+1}, \ldots, p_{t-1}$ belonging to $A^{-}$are two odd numbers and $\left(\frac{\pi_{1}^{\prime}}{\pi_{2}^{\prime}}\right)=-1$.

Let $F=\mathbb{Q}\left(\sqrt{-p_{1} p_{2}}\right)$ be an imaginary quadratic field with $p_{1} \equiv p_{2} \equiv 1 \bmod$ 4. By Rédei's criterion, we have that $r_{4}(C(F))=2$ if and only if $p_{1} \equiv p_{2} \equiv$ $1 \bmod 8$ and $\left(\frac{p_{1}}{p_{2}}\right)=1$. By Theorem 3.6 and Lemma 2.2, we have proved:

Corollary 3.7. Let $F=\mathbb{Q}\left(\sqrt{-p_{1} p_{2}}\right)$ be an imaginary quadratic field with primes $p_{1} \equiv p_{2} \equiv 1 \bmod 8$ and $\left(\frac{p_{1}}{p_{2}}\right)=1$. Let $\mathfrak{p}_{1}^{2}=p_{1} \mathcal{O}_{F}$ and $\mathfrak{t}^{2}=2 \mathcal{O}_{F}$. Then

(i) $\left[\mathfrak{p}_{1}\right] \in C(F)^{4}$ if and only if $\left(\frac{p_{1}}{p_{2}}\right)_{4}=\left(\frac{p_{2}}{p_{1}}\right)_{4}=1$.

(ii) $[\mathfrak{t}] \in C(F)^{4}$ if and only if $\left(\frac{\pi_{1}}{\pi_{2}}\right)=\left(\frac{1-\sqrt{2}}{\pi_{1}}\right)=\left(\frac{1-\sqrt{2}}{\pi_{2}}\right)$ if and only if either $p_{1}, p_{2} \in A^{+}$and $\left(\frac{\pi_{1}}{\pi_{2}}\right)=1$, or $p_{1}, p_{2} \in A^{-}$and $\left(\frac{\pi_{1}}{\pi_{2}}\right)=-1$ if and only if either $p_{1}, p_{2} \in A^{+}$and $p_{2}^{h_{+}\left(2 p_{1}\right) / 4}=x^{2}-2 p_{1} y^{2}$ for some $x, y \in \mathbb{Z}$, or $p_{1}, p_{2} \in A^{-}$and $\pm p_{2}^{h_{+}\left(2 p_{1}\right) / 4}=2 x^{2}-p_{1} y^{2}$ for some $x, y \in \mathbb{Z}$, where $\pi_{1}$ and $\pi_{2}$ are defined as in $\S 2$. Moreover, $r_{8}(C(F))=2$ if and only if $\left[\mathfrak{p}_{1}\right],[\mathfrak{t}] \in C(F)^{4}$ if and only if $\left(\frac{p_{1}}{p_{2}}\right)_{4}=\left(\frac{p_{2}}{p_{1}}\right)_{4}=1$ and $\left(\frac{\pi_{1}}{\pi_{2}}\right)=\left(\frac{1-\sqrt{2}}{\pi_{1}}\right)=\left(\frac{1-\sqrt{2}}{\pi_{2}}\right)$.

We now turn to another imaginary quadratic fields $F=\mathbb{Q}(\sqrt{-2 d})$ with $d=$ $p_{1} \cdots p_{t-1}$ and distinct primes $p_{i} \equiv 1 \bmod 4$. We know that $r_{2}(C(F))=t-1$ by genus theory and the Rédei's matrix $R_{F}$ is a symmetric matrix. We have that $r_{4}(C(F))=1$ if and only if $\operatorname{rank} R_{F}=t-2$ and $D^{*}(F)=\left\{1, q_{1}, 2 q_{2}, 2 d\right\}$, where $q_{1}=p_{1} \cdots p_{r}$ and $q_{2}=p_{r+1} \cdots p_{t-1}$.

Theorem 3.8. Let $F=\mathbb{Q}(\sqrt{-2 d})$ be an imaginary quadratic field with $d=$ $p_{1} \cdots p_{t-1}$ and distinct primes $p_{i} \equiv 1 \bmod 4$. Let $\operatorname{rank} R_{F}=t-2$ and $D^{*}(F)=$ $\left\{1, q_{1}, 2 q_{2}, 2 d\right\}$. 
(i) Suppose $q_{1}=p_{1} \cdots p_{r} \equiv 1 \bmod 8, q_{2}=p_{r+1} \cdots p_{t-1}$ and $1 \leq r<t-1$. Then $r_{8}(C(F))=1$ if and only if $\left(\frac{2 q_{2}}{q_{1}}\right)_{4}=1$.

(ii) Suppose $p_{i} \equiv 1 \bmod 8$ for $1 \leq i \leq t-1$, that is, $q_{1}=d$ and $q_{2}=1$. Then $r_{8}(C(F))=1$ if and only if an even number of the primes $p_{1}, \ldots, p_{t-1}$ belong to $B^{-}$.

Proof. (i) Suppose $\operatorname{rank} R_{F}=t-2$ and $q_{1}=p_{1} \cdots p_{r} \in D(F)$. Then the sum of the first $r$ row vectors of $R_{F}$ is equal to zero. Let $z^{2}=q_{1} x^{2}+2 q_{2} y^{2}$ have a relatively prime solution $(x, y, z)=(a, b, c)$ over $\mathbb{N}$. Then $\left[\mathfrak{q}_{1}\right]=\left[\mathfrak{p}_{c}\right]^{2} \in C(F)^{2}$, where $\mathfrak{q}_{1}^{2}=q_{1} \mathcal{O}_{F}$ and $\mathfrak{p}_{c}$ is an ideal of $F$ over $c$. Since $c^{2}=q_{1} a^{2}+2 q_{2} b^{2}$ and $q_{1} \equiv 1 \bmod 8$, we have that $\left(\frac{b}{q_{1}}\right)=1$ and $\left(\frac{c}{q_{1}}\right)=\left(\frac{2 q_{2}}{q_{1}}\right)_{4}$. Similarly, we conclude that

$$
r_{8}(C(F))=1 \Leftrightarrow\left[\mathfrak{q}_{1}\right] \in C(F)^{4} \Leftrightarrow\left(\frac{c}{q_{1}}\right)=\left(\frac{2 q_{2}}{q_{1}}\right)_{4}=1 .
$$

(ii) Let $\mathfrak{t}^{2}=2 \mathcal{O}_{F}$. Then by the process of proving (i), we conclude that $r_{8}(C(F))=1 \Leftrightarrow[\mathfrak{t}] \in C(F)^{4} \Leftrightarrow\left(\frac{2}{p_{1} \cdots p_{t-1}}\right)_{4}=1 \Leftrightarrow$ an even number of the primes $p_{1}, \ldots, p_{t-1}$ belong to $B^{-}$.

Let $F=\mathbb{Q}\left(\sqrt{-2 p_{1} p_{2}}\right)$ be an imaginary quadratic field with $p_{1} \equiv p_{2} \equiv$ 1 mod 4. By Rédei's criterion, we have that $r_{4}(C(F))=1$ if and only if one of the following conditions holds:

(1) $p_{1} \equiv p_{2}+4 \equiv 1 \bmod 8$ and $\left(\frac{p_{1}}{p_{2}}\right)=1$;

(2) $p_{1} \equiv p_{2} \equiv 1 \bmod 8$ and $\left(\frac{p_{1}}{p_{2}}\right)=-1$.

By Theorem 3.8, we get:

Corollary 3.9. Let $F=\mathbb{Q}\left(\sqrt{-2 p_{1} p_{2}}\right)$ be an imaginary quadratic field.

(i) Suppose $p_{1} \equiv p_{2}+4 \equiv 1 \bmod 8$ and $\left(\frac{p_{1}}{p_{2}}\right)=1$. Then $r_{8}(C(F))=1$ if and only if $\left(\frac{2 p_{2}}{p_{1}}\right)_{4}=1$.

(ii) Suppose $p_{1} \equiv p_{2} \equiv 1 \bmod 8$ and $\left(\frac{p_{1}}{p_{2}}\right)=-1$. Then $r_{8}(C(F))=1$ if and only if $\left(\frac{2}{p_{1} p_{2}}\right)_{4}=1$ if and only if either $p_{1}, p_{2} \in B^{+}$or $p_{1}, p_{2} \in B^{-}$.

Example 3.10. In Corollary 3.9, let $F=\mathbb{Q}\left(\sqrt{-2 p_{1} p_{2}}\right)$ with distinct primes $p_{1} \equiv p_{2} \equiv 1 \mathrm{mod} 4$. Let $C(F)_{2}$ denote the 2-primary subgroup of $C(F)$.

(i) For $p_{1}=17$ and $p_{2}=53,\left(\frac{53}{17}\right)=\left(\frac{2}{17}\right)=1,\left(\frac{2 p_{2}}{p_{1}}\right)_{4}=\left(\frac{2 \cdot 53}{17}\right)_{4}=\left(\frac{4}{17}\right)_{4}=$ 1 , so $r_{8}(C(F))=1$ by Corollary 3.9(i). In fact, $C(F)_{2} \cong \mathbb{Z} /(16) \oplus \mathbb{Z} /(2)$ by Pari-GP.

(ii) For $p_{1}=17$ and $p_{2}=97,\left(\frac{97}{17}\right)=\left(\frac{12}{17}\right)=-1$ and $17,97 \in B^{-}$, so $r_{8}(C(F))=1$ by Corollary 3.9(ii). In fact, $C(F)_{2} \cong \mathbb{Z} /(8) \oplus \mathbb{Z} /(2)$ by Pari-GP.

Let $F=\mathbb{Q}(\sqrt{-2 d})$ be an imaginary quadratic field with $d=p_{1} \cdots p_{t-1}$ and distinct primes $p_{i} \equiv 1 \bmod 8$. Then the Rédei's matrix is

$$
R_{F}=\left(\begin{array}{cc}
M & 0 \\
0 & 0
\end{array}\right) \text {, }
$$


where the $(t-1) \times(t-1)$ matrix $M$ is equal to the Rédei's matrix $R_{E}$ of $E=\mathbb{Q}(\sqrt{d})$. Let $p_{i}=u_{i}^{2}-2 w_{i}^{2}$ and $\pi_{i}=u_{i}+w_{i} \sqrt{2}$ for $1 \leq i \leq t-1$.

Theorem 3.11. Let $F=\mathbb{Q}(\sqrt{-2 d})$ be an imaginary quadratic field with $d=$ $p_{1} \cdots p_{t-1}$ and distinct primes $p_{i} \equiv 1 \bmod 8$. Suppose rank $R_{F}=t-3$, that is, $D(F)=(2) \times\left(q_{1}\right) \times(2 d)$, where $q_{1}=p_{1} \cdots p_{r}$ and $q_{2}=p_{r+1} \cdots p_{t-1}$. Let $\mathfrak{q}_{1}^{2}=$ $q_{1} \mathcal{O}_{F}, \mathfrak{t}^{2}=2 \mathcal{O}_{F}, \pi_{1}^{\prime}=\prod_{i=1}^{r} \pi_{i}=u_{1}^{\prime}+w_{1}^{\prime} \sqrt{2}$ and $\pi_{2}^{\prime}=\prod_{i=r+1}^{t-1} \pi_{i}=u_{2}^{\prime}+w_{2}^{\prime} \sqrt{2}$. Then we have

(i) $[\mathfrak{t}] \in C(F)^{4}$ if and only if $\left(\frac{2}{q_{1}}\right)_{4}=\left(\frac{2}{q_{2}}\right)_{4}=\left(\frac{\pi_{2}^{\prime}}{\pi_{1}^{\prime}}\right)$ if and only if either both $p_{1}, \ldots, p_{r}$ and $p_{r+1}, \ldots, p_{t-1}$ belonging to $B^{-}$are two even numbers and $\left(\frac{\pi_{1}^{\prime}}{\pi_{2}^{\prime}}\right)=1$ or both $p_{1}, \ldots, p_{r}$ and $p_{r+1}, \ldots, p_{t-1}$ belonging to $B^{-}$are two odd numbers and $\left(\frac{\pi_{1}^{\prime}}{\pi_{2}^{\prime}}\right)=-1$.

(ii) $\left[\mathfrak{q}_{1}\right] \in C(F)^{4}$ if and only if $\left(\frac{2 q_{2}}{q_{1}}\right)_{4}=\left(\frac{q_{1}}{q_{2}}\right)_{4}\left(\frac{\pi_{1}^{\prime}}{\pi_{2}^{\prime}}\right)=1$.

Proof. (i) By the assumption, we know that the two sums of both the first $r$ row vectors and the first $t-1$ row vectors of $R_{F}$ are equal to zero. Since $d=q_{1} q_{2}=u^{2}-2 w^{2}$, where $u=u_{1}^{\prime} u_{2}^{\prime}+2 w_{1}^{\prime} w_{2}^{\prime}$ and $w=u_{1}^{\prime} w_{2}^{\prime}+u_{2}^{\prime} w_{1}^{\prime}$, $2 u^{2}=4 w^{2}+2 d$ and $[\mathfrak{t}]=\left[\mathfrak{p}_{u}\right]^{2} \in C(F)^{2}$, where $\mathfrak{p}_{u}$ is an ideal of $F$ over $u$. Similarly, we conclude that

$$
[\mathfrak{t}] \in C(F)^{4} \Leftrightarrow\left(\frac{u}{q_{1}}\right)=\left(\frac{u}{q_{2}}\right)=1 .
$$

On the other hand, for each $p_{i}(1 \leq i \leq r), \mathcal{O}_{L} /\left(\pi_{i}\right) \cong \mathbb{Z} /\left(p_{i}\right), u=u_{1}^{\prime} u_{2}^{\prime}+$ $2 w_{1}^{\prime} w_{2}^{\prime} \equiv u_{1}^{\prime}\left(u_{2}^{\prime}-w_{2}^{\prime} \sqrt{2}\right) \bmod \left(\pi_{i}\right)$ and $\left(\frac{\pi_{2}^{\prime}}{\pi_{i}}\right)=\left(\frac{u_{2}^{\prime}-w_{2}^{\prime} \sqrt{2}}{\pi_{i}}\right)$ since $\left(\frac{q_{2}}{p_{i}}\right)=\left(\frac{q_{2}}{\pi_{i}}\right)=1$. Then

$$
\left(\frac{u}{p_{i}}\right)=\left(\frac{u}{\pi_{i}}\right)=\left(\frac{u_{1}^{\prime}}{\pi_{i}}\right)\left(\frac{\pi_{2}^{\prime}}{\pi_{i}}\right)=\left(\frac{u_{1}^{\prime}}{p_{i}}\right)\left(\frac{\pi_{2}^{\prime}}{\pi_{i}}\right) .
$$

Similarly, for each $p_{j}(r+1 \leq j \leq t-1)$,

$$
\left(\frac{u}{p_{j}}\right)=\left(\frac{u}{\pi_{j}}\right)=\left(\frac{u_{2}^{\prime}}{\pi_{j}}\right)\left(\frac{\pi_{1}^{\prime}}{\pi_{j}}\right)=\left(\frac{u_{2}^{\prime}}{p_{j}}\right)\left(\frac{\pi_{1}^{\prime}}{\pi_{j}}\right) .
$$

Since $q_{1}=u_{1}^{\prime 2}-2 w_{1}^{\prime 2}$, we have that $\left(\frac{w_{1}^{\prime}}{q_{1}}\right)=1$ and $\left(\frac{2}{q_{1}}\right)_{4}=\left(\frac{u_{1}^{\prime}}{q_{1}}\right)$, similarly, $\left(\frac{2}{q_{2}}\right)_{4}=\left(\frac{u_{2}^{\prime}}{q_{2}}\right)$. By reciprocity law, $\left(\frac{\pi_{1}^{\prime}}{\pi_{2}^{\prime}}\right)=\left(\frac{\pi_{2}^{\prime}}{\pi_{1}^{\prime}}\right)$. Hence we conclude that $[\mathfrak{t}] \in C(F)^{4} \Leftrightarrow\left(\frac{2}{q_{1}}\right)_{4}=\left(\frac{2}{q_{2}}\right)_{4}=\left(\frac{\pi_{2}^{\prime}}{\pi_{1}^{\prime}}\right) \Leftrightarrow$ either both $p_{1}, \ldots, p_{r}$ and $p_{r+1}, \ldots, p_{t-1}$ belonging to $B^{-}$are two even numbers and $\left(\frac{\pi_{1}^{\prime}}{\pi_{2}^{\prime}}\right)=1$ or both $p_{1}, \ldots, p_{r}$ and $p_{r+1}, \ldots, p_{t-1}$ belonging to $B^{-}$are two odd numbers and $\left(\frac{\pi_{1}^{\prime}}{\pi_{2}^{\prime}}\right)=-1$.

(ii) Let $z^{2}=q_{1} x^{2}+2 q_{2} y^{2}$, where $q_{1}=p_{1} \cdots p_{r}$ and $q_{2}=d / q_{1}$, have a relatively prime solution $(x, y, z)=(a, b, c)$ over $\mathbb{N}$. Then $\left[\mathfrak{q}_{1}\right]=\left[\mathfrak{p}_{c}\right]^{2} \in C(F)^{2}$, where $\mathfrak{q}_{1}^{2}=q_{1} \mathcal{O}_{F}$ and $\mathfrak{p}_{c}$ is an ideal of $F$ over $c$. Since $c^{2}=q_{1} a^{2}+2 q_{2} b^{2}$, we 
have that $\left(\frac{b}{q_{1}}\right)=1$ and $\left(\frac{c}{q_{1}}\right)=\left(\frac{2 q_{2}}{q_{1}}\right)_{4},\left(\frac{c}{q_{2}}\right)=\left(\frac{q_{1}}{q_{2}}\right)_{4}\left(\frac{a}{q_{2}}\right)$. Similarly, we have that

$$
\left[\mathfrak{q}_{1}\right] \in C(F)^{4} \Leftrightarrow\left(\frac{c}{q_{1}}\right)=\left(\frac{c}{q_{2}}\right)=1 .
$$

We need to determine the value of the Jacobi symbol $\left(\frac{a}{q_{1}}\right)$. Let $2 u^{2}=4 w^{2}+2 d$ and $q_{1} c^{2}=\left(q_{1} a\right)^{2}+2 d b^{2}$. Then $2 q_{1} u^{2} c^{2}=N_{F / \mathbb{Q}}\left(q_{1} a+b \sqrt{-2 d}\right) N_{F / \mathbb{Q}}(2 w+$ $\sqrt{-2 d})$, i.e.,

$$
2 q_{1} u^{2} c^{2}=4 q_{1}^{2}\left(a w-q_{2} b\right)^{2}+2 d\left(q_{1} a+2 b w\right)^{2} .
$$

We can choose a solution $(x, y, z)=(a, b, c)$ of the equation $z^{2}=q_{1} x^{2}+2 q_{2} y^{2}$ such that the greatest common divisor $\left(u c, a w-q_{2} b\right)=1$. In fact, in $F=$ $\mathbb{Q}(\sqrt{-2 d})$, let $\mathfrak{t p}_{u}^{2}=(2 w+\sqrt{-2 d}) \mathcal{O}_{F}$, where $\mathfrak{t}$ is the dyadic ideal of $F$ and $\mathfrak{p}_{u}$ is an ideal of $F$ over $u$. Since $\left[\mathfrak{q}_{1}\right] \in C(F)^{2}$, there is an ideal $\mathfrak{p}_{c}$ of $F$ over positive integer number $c$ such that $\left[\mathfrak{q}_{1}\right]\left[\mathfrak{p}_{c}\right]^{2}=1$ and $\mathfrak{p}_{c}+\overline{\mathfrak{p}}_{c}=\mathcal{O}_{F}=\mathfrak{p}_{u}+\overline{\mathfrak{p}}_{c}$, where $\overline{\mathfrak{p}}_{c}$ is the conjugate ideal of $\mathfrak{p}_{c}$. Hence $\mathfrak{q}_{1} \mathfrak{p}_{c}^{2}=(a+b \sqrt{-2 d}) \mathcal{O}_{F}$ and we get such $(x, y, z)=(a, b, c)$ satisfying $\left(u c, a w-q_{2} b\right)=1$.

By $(3.2)$, we have the Jacobi symbol $\left(\frac{a w-q_{2} b}{q_{2}}\right)=\left(\frac{a w}{q_{2}}\right)=1$, i.e., $\left(\frac{a}{q_{2}}\right)=\left(\frac{w}{q_{2}}\right)$. On the other hand,

$$
\begin{aligned}
q_{1} q_{2} & =N_{L / \mathbb{Q}}\left(u_{1}^{\prime}+w_{1} \sqrt{2}\right) N_{L / \mathbb{Q}}\left(u_{2}^{\prime}+w_{2}^{\prime} \sqrt{2}\right) \\
& =\left(u_{1}^{\prime} u_{2}^{\prime}+2 w_{1}^{\prime} w_{2}^{\prime}\right)^{2}-2\left(u_{1}^{\prime} w_{2}^{\prime}+u_{2}^{\prime} w_{1}^{\prime}\right)^{2}=u^{2}-2 w^{2},
\end{aligned}
$$

where $u=u_{1}^{\prime} u_{2}^{\prime}+2 w_{1}^{\prime} w_{2}^{\prime}$ and $w=u_{1}^{\prime} w_{2}^{\prime}+u_{2}^{\prime} w_{1}^{\prime}$. For each $p_{j}(r+1 \leq j \leq t-1)$, $\mathcal{O}_{L} /\left(\pi_{j}\right) \cong \mathbb{Z} /\left(p_{j}\right), w=u_{1}^{\prime} w_{2}+u_{2}^{\prime} w_{1}^{\prime} \equiv w_{2}^{\prime}\left(u_{1}^{\prime}-w_{1} \sqrt{2}\right) \bmod \left(\pi_{j}\right)$. Hence

$$
\left(\frac{w}{p_{j}}\right)=\left(\frac{w}{\pi_{j}}\right)=\left(\frac{w_{2}^{\prime}}{\pi_{j}}\right)\left(\frac{u_{1}^{\prime}-w_{1}^{\prime} \sqrt{2}}{\pi_{j}}\right)=\left(\frac{w_{2}^{\prime}}{p_{j}}\right)\left(\frac{u_{1}^{\prime}-w_{1}^{\prime} \sqrt{2}}{\pi_{j}}\right) .
$$

Since $q_{2}=u_{2}^{\prime 2}-2 w_{2}^{\prime 2}$, the Jacobi symbol $\left(\frac{w_{2}^{\prime}}{q_{2}}\right)=1$; by $\left(\frac{q_{1}}{q_{2}}\right)=1,\left(\frac{\pi_{1}^{\prime}}{\pi_{2}^{\prime}}\right)=$ $\left(\frac{u_{1}^{\prime}-w_{1}^{\prime} \sqrt{2}}{\pi_{2}^{\prime}}\right)$. Hence $\left(\frac{a}{q_{2}}\right)=\left(\frac{w}{q_{2}}\right)=\left(\frac{\pi_{1}^{\prime}}{\pi_{2}}\right)$. As a conclusion, we get that

$$
\left[\mathfrak{q}_{1}\right] \in C(F)^{4} \Leftrightarrow\left(\frac{2 q_{2}}{q_{1}}\right)_{4}=\left(\frac{q_{1}}{q_{2}}\right)_{4}\left(\frac{\pi_{1}^{\prime}}{\pi_{2}^{\prime}}\right)=1 .
$$

Let $F=\mathbb{Q}\left(\sqrt{-2 p_{1} p_{2}}\right)$ be an imaginary quadratic field with distinct primes $p_{1} \equiv p_{2} \equiv 1 \bmod 4$. By Rédei's criterion, we have that $r_{4}(C(F))=2$ if and only if $p_{1} \equiv p_{2} \equiv 1 \bmod 8$ and $\left(\frac{p_{1}}{p_{2}}\right)=1$. By Theorem 3.11 and Lemma 2.2, we get:

Corollary 3.12. Let $F=\mathbb{Q}\left(\sqrt{-2 p_{1} p_{2}}\right)$ be an imaginary quadratic field with district primes $p_{1} \equiv p_{2} \equiv 1 \bmod 8$ and $\left(\frac{p_{1}}{p_{2}}\right)=1$. Let $\mathfrak{t}^{2}=2 \mathcal{O}_{F}$ and $\mathfrak{p}_{1}^{2}=p_{1} \mathcal{O}_{F}$. Then

(i) $[\mathfrak{t}] \in C(F)^{4}$ if and only if $\left(\frac{2}{p_{1}}\right)_{4}=\left(\frac{2}{p_{2}}\right)_{4}=\left(\frac{\pi_{1}}{\pi_{2}}\right)$ if and only if either $p_{1}, p_{2} \in B^{+}, p_{2}^{h_{+}\left(2 p_{1}\right) / 4}=x^{2}-2 p_{1} y^{2}$ over $\mathbb{Z}$ or $p_{1}, p_{2} \in B^{-}, \pm p_{2}^{h_{+}}\left(2 p_{1}\right) / 4$ $=2 x^{2}-p_{1} y^{2}$ over $\mathbb{Z}$. 
(ii) $\left[\mathfrak{p}_{1}\right] \in C(F)^{4}$ if and only if $\left(\frac{2 p_{2}}{p_{1}}\right)_{4}=\left(\frac{p_{1}}{p_{2}}\right)_{4} \cdot\left(\frac{\pi_{1}}{\pi_{2}}\right)=1$. Moreover, $r_{8}(C(F))=2$ if and only if $\left(\frac{p}{q}\right)_{4}=\left(\frac{q}{p}\right)_{4}=\left(\frac{2}{p}\right)_{4}=\left(\frac{2}{q}\right)_{4}=\left(\frac{\pi_{1}}{\pi_{2}}\right)$.

Example 3.13. Let $F=\mathbb{Q}(\sqrt{-2 \cdot 41 \cdot 241}),\left(\frac{241}{41}\right)=1$. Then $C(F)_{2} \cong \mathbb{Z} /(8) \oplus$ $\mathbb{Z} /(8)$ by Pari-GP. We also verify the condition of Corollary 3.12 . It is clear that $41=3^{2}+32,41 \in A^{+}, 41,241 \in B^{-}$and $\left(\frac{241}{41}\right)_{4}=\left(\frac{36}{41}\right)_{4}=\left(\frac{2}{41}\right)\left(\frac{3}{41}\right)=-1$. In terms of norm from $\mathbb{Q}(\sqrt{-1}), 41=5^{2}+4^{2}, 241=15^{2}+4^{2},\left(\frac{41}{241}\right)_{4}\left(\frac{241}{41}\right)_{4}=$ $(-1)^{\frac{41-1}{4}}\left(\frac{15 \cdot 4-15 \cdot 4}{41}\right)=1$ by quartic reciprocity. So $\left(\frac{41}{241}\right)_{4}=-1$. By $41=$ $13^{2}-2 \cdot 8^{2}, 241=29^{2}-2 \cdot 20^{2}$, let $\pi_{1}=13-8 \sqrt{2}$ and $\pi_{2}=29-20 \sqrt{2}$. Then $\left(\frac{\pi_{2}}{\pi_{1}}\right)=\left(\frac{29 \cdot 2-40 \sqrt{2}}{13-8 \sqrt{2}}\right)\left(\frac{2}{13-8 \sqrt{2}}\right)=\left(\frac{-7 \cdot 2}{41}\right)=-1$. Hence, the 8-rank of $C(F)$ is equal to 2 by Corollary 3.12 .

\section{Densities}

In the section, we use a Gerth's method (see $[4,5,6,16]$ ) to investigate the densities of 8-rank of $C(F)$ equal to 1 or 2 in all quadratic number fields $F=\mathbb{Q}\left(\sqrt{-\varepsilon p_{1} p_{2}}\right)$, where $\varepsilon \in\{1,2\}$ and $p_{1} \equiv p_{2} \equiv 1 \bmod 4$. For a positive real number $x$, let

$A_{x}=\left\{p_{1} p_{2}:\right.$ distinct primes $p_{1} \equiv p_{2} \equiv 1 \bmod 4, p_{1}<p_{2}$ and $\left.p_{1} p_{2} \leq x\right\}$,

$A_{1, x}=\left\{F=\mathbb{Q}\left(\sqrt{-p_{1} p_{2}}\right): r_{4}(C(F))=r_{8}(C(F))=1\right.$ and $\left.p_{1} p_{2} \in A_{x}\right\}$,

$A_{2, x}=\left\{F=\mathbb{Q}\left(\sqrt{-p_{1} p_{2}}\right): r_{4}(C(F))=r_{8}(C(F))=2\right.$ and $\left.p_{1} p_{2} \in A_{x}\right\}$,

$A_{3, x}=\left\{F=\mathbb{Q}\left(\sqrt{-2 p_{1} p_{2}}\right): r_{4}(C(F))=r_{8}(C(F))=1\right.$ and $\left.p_{1} p_{2} \in A_{x}\right\}$,

$A_{4, x}=\left\{F=\mathbb{Q}\left(\sqrt{-2 p_{1} p_{2}}\right): r_{4}(C(F))=r_{8}(C(F))=2\right.$ and $\left.p_{1} p_{2} \in A_{x}\right\}$.

We define densities $d_{i}(1 \leq i \leq 4)$ as follows:

$$
d_{i}=\lim _{x \rightarrow \infty} \frac{\left|A_{i, x}\right|}{\left|A_{x}\right|} .
$$

Theorem 4.1. Let $d_{1}, d_{2}, d_{3}$ and $d_{4}$ be defined as (4.1). Then

$$
d_{1}=\frac{5}{16}, d_{2}=\frac{1}{128}, d_{3}=\frac{3}{16}, d_{4}=\frac{1}{128} .
$$

Proof. We know that, by ([7, Theorem 437]) and $p_{1} \equiv p_{2} \equiv 1 \bmod 4, p_{1}<p_{2}$,

$$
\left|A_{x}\right|=\sum_{p_{1} p_{2} \in A_{x}} 1=\frac{x \log \log x}{4 \log x}+o\left(\frac{x \log \log x}{\log x}\right) .
$$

Let $F=\mathbb{Q}\left(\sqrt{-p_{1} p_{2}}\right) \in A_{1, x}$. Then by Corollary 3.4, we have that $r_{4}(C(F))=$ $r_{8}(C(F))=1$ if and only if one of the following five conditions holds:

(1) $p_{1} \equiv p_{2}+4 \equiv 1 \bmod 8,\left(\frac{p_{2}}{p_{1}}\right)=1$ and $\left(\frac{p_{2}}{p_{1}}\right)_{4}=1$;

(2) $p_{1}+4 \equiv p_{2} \equiv 1 \bmod 8,\left(\frac{p_{2}}{p_{1}}\right)=1$ and $\left(\frac{p_{1}}{p_{2}}\right)_{4}=1$;

(3) $p_{1} \equiv p_{2} \equiv 5 \bmod 8,\left(\frac{p_{2}}{p_{1}}\right)=1$ and $\left(\frac{\lambda_{2}}{\lambda_{1}}\right)=1$, where $\lambda_{1}, \lambda_{2}$ are defined as Lemma 2.3; 
8-RANKS OF CLASS GROUPS OF IMAGINARY QUADRATIC NUMBER FIELDS 1265

(4) $p_{1} \equiv p_{2} \equiv 5 \bmod 8,\left(\frac{p_{2}}{p_{1}}\right)=-1$ and $\left(\frac{\lambda_{2}}{\lambda_{1}}\right)=1$, where $\lambda_{1}, \lambda_{2}$ are defined as Lemma 2.3;

(5) $p_{1} \equiv p_{2} \equiv 1 \bmod 8,\left(\frac{p_{2}}{p_{1}}\right)=-1$ and $\left(\frac{1-\sqrt{2}}{\pi_{1} \pi_{2}}\right)=1$, where $\pi_{1}, \pi_{2}$ are defined as $\S 2$.

Hence

$$
\begin{aligned}
\left|A_{1, x}(F)\right|= & \sum_{\substack{p_{1} p_{2} \in A_{x} \\
p_{1} \equiv p_{2}+4 \equiv 1 \bmod 8}} \frac{1}{4}\left(1+\left(\frac{p_{2}}{p_{1}}\right)\right)\left(1+\left(\frac{p_{2}}{p_{1}}\right)_{4}\right) \\
& +\sum_{\substack{p_{1} p_{2} \in A_{x} \\
p_{1}+4 \equiv p_{2} \equiv \text { mod } 8}} \frac{1}{4}\left(1+\left(\frac{p_{2}}{p_{1}}\right)\right)\left(1+\left(\frac{p_{1}}{p_{2}}\right)_{4}\right) \\
& +\sum_{\substack{p_{1} p_{2} \in A_{x} \\
p_{1} \equiv p_{2} \equiv 5 \bmod 8}} \frac{1}{4}\left(1+\left(\frac{p_{2}}{p_{1}}\right)\right)\left(1+\left(\frac{\lambda_{2}}{\lambda_{1}}\right)\right) \\
& +\sum_{\substack{p_{1} p_{2} \in A_{x} \\
p_{1} \equiv p_{2} \equiv 5 \bmod 8}} \frac{1}{4}\left(1-\left(\frac{p_{2}}{p_{1}}\right)\right)\left(1+\left(\frac{\lambda_{2}}{\lambda_{1}}\right)\right) \\
& +\sum_{\substack{p_{1} p_{2} \in A_{x} \\
p_{1} \equiv p_{2} \equiv \equiv \bmod 8}} \frac{1}{4}\left(1-\left(\frac{p_{2}}{p_{1}}\right)\right)\left(1+\left(\frac{1-\sqrt{2}}{\pi_{1} \pi_{2}}\right)\right) \\
= & \sum_{p q \in A_{x}}\left(\frac{1}{16}+\frac{1}{16}+\frac{1}{16}+\frac{1}{16}+\frac{1}{16}\right)+o\left(\frac{x \log \log x}{\log x}\right) \\
= & \frac{5}{64} \cdot \frac{x \log \log x}{\log x}+o\left(\frac{x \log \log x}{\log x}\right) .
\end{aligned}
$$

An intuitive explanation of the formula might proceed as follows. In the second equation, a factor of $\frac{1}{4}$ is introduced by each congruence relation of $p_{1}, p_{2} \bmod 8$. This is considered in detail in $[4,6]$.

For the sake of completeness, we give a sketch of proof.

$$
\begin{aligned}
& \sum_{\substack{p_{1} p_{2} \in A_{x} \\
p_{1} \equiv p_{2}+4 \equiv 1 \bmod 8}} \frac{1}{4}\left(1+\left(\frac{p_{2}}{p_{1}}\right)\right)\left(1+\left(\frac{p_{2}}{p_{1}}\right)_{4}\right) \\
= & \frac{1}{16} \sum_{p_{1} p_{2} \in A_{x}} 1+O\left(\sum_{\substack{p_{1} p_{2} \in A_{x} \\
p_{1} \equiv p_{2}+4 \equiv 1 \bmod 8}}\left(\chi_{1}\left(p_{2}\right)+\chi_{2}\left(p_{2}\right)+\chi_{3}\left(p_{2}\right)\right)\right) \\
= & \frac{x \log \log x}{64 \log x}+o\left(\frac{x \log \log x}{\log x}\right),
\end{aligned}
$$

where $\chi_{1}\left(p_{2}\right)=\left(\frac{p_{2}}{p_{1}}\right), \chi_{2}\left(p_{2}\right)=\left(\frac{p_{2}}{p_{1}}\right)_{4}, \chi_{3}\left(p_{3}\right)=\left(\frac{p_{2}}{p_{1}}\right)_{4}\left(\frac{p_{2}}{p_{1}}\right)$ are Dirichlet characters modulo $p_{1}$. By $[6$, Theorem 2$]$, we have that

$$
\sum \chi_{i}\left(p_{2}\right)=o\left(\frac{x \log \log x}{\log x}\right) \quad \text { for } i=1,2,3 \text {. }
$$


Similarly, we have above character sum estimate for the product of characters: $\left(\frac{p_{2}}{p_{1}}\right),\left(\frac{p_{2}}{p_{1}}\right)_{4},\left(\frac{\lambda_{2}}{\lambda_{1}}\right),\left(\frac{1-\sqrt{2}}{\pi_{1} \pi_{2}}\right)$.

\section{Hence}

$$
d_{1}=\lim _{x \rightarrow \infty} \frac{\left|A_{1, x}\right|}{\left|A_{x}\right|}=\frac{5}{16} .
$$

Let $F=\mathbb{Q}\left(\sqrt{-p_{1} p_{2}}\right) \in A_{2, x}$. Then, by Corollary 3.7, we have that $r_{4}(C(F))$ $=r_{8}(C(F))=2$ if and only if $p_{1} \equiv p_{2} \equiv 1 \bmod 8,\left(\frac{p_{1}}{p_{2}}\right)_{4}=\left(\frac{p_{2}}{p_{1}}\right)_{4}=1$ and $\left(\frac{\pi_{1}}{\pi_{2}}\right)=\left(\frac{1-\sqrt{2}}{\pi_{1}}\right)=\left(\frac{1-\sqrt{2}}{\pi_{2}}\right)$. Hence

$$
\begin{aligned}
\left|A_{2, x}(F)\right|= & \sum_{\substack{p_{1} p_{2} \in A_{x} \\
p_{1} \equiv p_{2} \equiv 1 \bmod 8}} \frac{1}{32}\left(1+\left(\frac{p_{2}}{p_{1}}\right)\right)\left(1+\left(\frac{p_{2}}{p_{1}}\right)_{4}\right)\left(1+\left(\frac{p_{1}}{p_{2}}\right)_{4}\right) \\
& \times\left(1+\left(\frac{\pi_{1}(1-\sqrt{2})}{\pi_{2}}\right)\right)\left(1+\left(\frac{1-\sqrt{2}}{\pi_{1} \pi_{2}}\right)\right) \\
& =\sum_{\substack{p_{1} p_{2} \in A_{x} \\
p_{1} \equiv p_{2} \equiv 1 \bmod 8}} \frac{1}{32}+o\left(\frac{x \log \log x}{\log x}\right) \\
& =\frac{x \log \log x}{512 \log x}+o\left(\frac{x \log \log x}{\log x}\right) .
\end{aligned}
$$

Thus

$$
d_{2}=\lim _{x \rightarrow \infty} \frac{\left|A_{2, x}\right|}{\left|A_{x}\right|}=\frac{1}{128} .
$$

Let $F=\mathbb{Q}\left(\sqrt{-2 p_{1} p_{2}}\right) \in A_{3, x}$. Then, by Corollary 3.9 , we have that $r_{4}(C(F))=r_{8}(C(F))=1$ if and only if one of the following three conditions holds:

(1) $p_{1} \equiv p_{2}+4 \equiv 1 \bmod 8,\left(\frac{p_{1}}{p_{2}}\right)=1$ and $\left(\frac{2 p_{2}}{p_{1}}\right)_{4}=1$;

(2) $p_{2} \equiv p_{1}+4 \equiv 1 \bmod 8,\left(\frac{p_{1}}{p_{2}}\right)=1$ and $\left(\frac{2 p_{1}}{p_{2}}\right)_{4}=1$;

(3) $p_{1} \equiv p_{2} \equiv 1 \bmod 8,\left(\frac{p_{1}}{p_{2}}\right)=-1$ and $\left(\frac{2}{p_{1} p_{2}}\right)_{4}=1$.

Hence

$$
\begin{aligned}
&\left|A_{3, x}\right|= \sum_{\substack{p_{1} p_{2} \in A_{x} \\
p_{1} \equiv p_{2}+4 \equiv 1 \bmod 8}} \frac{1}{4}\left(1+\left(\frac{p_{2}}{p_{1}}\right)\right)\left(1+\left(\frac{2 p_{2}}{p_{1}}\right)_{4}\right) \\
&+\sum_{\substack{p_{1} p_{2} \in A_{x} \\
p_{2} \equiv p_{1}+4 \equiv 1 \bmod 8}} \frac{1}{4}\left(1+\left(\frac{p_{1}}{p_{2}}\right)\right)\left(1+\left(\frac{2 p_{1}}{p_{2}}\right)_{4}\right) \\
&+\sum_{\substack{p_{1} p_{2} \in A_{x} \\
p_{1} \equiv p_{2} \equiv 1 \bmod 8}} \frac{1}{4}\left(1-\left(\frac{p_{1}}{p_{2}}\right)\right)\left(1+\left(\frac{2}{p_{1} p_{2}}\right)_{4}\right) \\
&=\sum_{p_{1} p_{2} \in A_{x}}\left(\frac{1}{16}+\frac{1}{16}+\frac{1}{16}\right)+o\left(\frac{x \log \log x}{\log x}\right)
\end{aligned}
$$




$$
=\frac{3}{64} \cdot \frac{x \log \log x}{\log x}+o\left(\frac{x \log \log x}{\log x}\right) .
$$

Hence

$$
d_{3}=\lim _{x \rightarrow \infty} \frac{\left|A_{3, x}\right|}{\left|A_{x}\right|}=\frac{3}{16} .
$$

Let $F=\mathbb{Q}\left(\sqrt{-2 p_{1} p_{2}}\right) \in A_{4, x}$. Then by Corollary 3.12, we have that $r_{4}(C(F))=r_{8}(C(F))=2$ if and only if $p_{1} \equiv p_{2} \equiv 1 \bmod 8,\left(\frac{p_{1}}{p_{2}}\right)_{4}=\left(\frac{p_{2}}{p_{1}}\right)_{4}=$ $\left(\frac{2}{p_{1}}\right)_{4}=\left(\frac{2}{p_{2}}\right)_{4}=\left(\frac{\pi_{1}}{\pi_{2}}\right)$. Hence

$$
\begin{aligned}
&\left|A_{4, x}\right|= \sum_{\substack{p_{1} p_{2} \in A_{x} \\
p_{1} \equiv p_{2} \equiv 1 \bmod 8}} \frac{1}{32}\left(1+\left(\frac{p_{1}}{p_{2}}\right)\right)\left(1+\left(\frac{2 p_{1}}{p_{2}}\right)_{4}\right)\left(1+\left(\frac{2 p_{2}}{p_{1}}\right)_{4}\right) \\
& \times\left(1+\left(\frac{2}{p_{1} p_{2}}\right)_{4}\right)\left(1+\left(\frac{2}{p_{1}}\right)_{4}\left(\frac{\pi_{1}}{\pi_{2}}\right)\right) \\
&=\frac{1}{512} \cdot \frac{x \log \log x}{\log x}+o\left(\frac{x \log \log x}{\log x}\right) .
\end{aligned}
$$

Hence

$$
d_{4}=\lim _{x \rightarrow \infty} \frac{\left|A_{4, x}\right|}{\left|A_{x}\right|}=\frac{1}{128} .
$$

\section{References}

[1] P. Barrucand and H. Cohn, Note on primes of type $x^{2}+32 y^{2}$, class number, and residuacity, J. Reine Angew. Math. 238 (1969), 67-70.

[2] P. E. Conner and J. Hurrelbrink, Class Number Parity, Ser. Pure Math. 8, Would Sci., Singapore 1988.

[3] — On the 4-rank of the tame kernel $K_{2}(\mathcal{O})$ in positive definite terms, J. Number Theory 88 (2001), no. 2, 263-282.

[4] F. Gerth III, Counting certain number fields with prescibed l-class numbers, J. Reine Angew. Math. 337 (1982), 195-207.

[5] - The 4-class ranks of quadratic fields, Invent. Math. 77 (1984), no. 3, 489-515.

[6] F. Gerth III and S. W. Graham, Application of a character sum estimate to a 2-class number density, J. Number Theory 19 (1984), no. 2, 239-247.

[7] G. Hardy and E. Wright, An Introduction to the Theory of Numbers, Fifth edition, London, 1979.

[8] E. Hecke, Lecture on the Theory of Algebraic Numbers, GTM 77, Springer-Verlag, 1981.

[9] J. Hurrelbrink and Q. Yue, On ideal class groups and units in terms of the quadratic form $x^{2}+32 y^{2}$, Chinese Ann. Math. Ser. B 26 (2005), no. 2, 239-252.

[10] K. Ireland and M. Rosen, A Classical Introduction to Modern Number Theory, GTM 84, Springer-Verlag, 1972

[11] J. Neukirch, Class Field Theory, Springer, Berlin, 1986.

[12] P. Stevenhagen, Divisibity by 2-powers of certain quadratic class numbers, J. Number Theory 43 (1993), no. 1, 1-19.

[13] X. Wu and Q. Yue, 8-ranks of class groups of some imaginary quadratic number fields, Acta Math. Sin. (Engl. Ser.) 23 (2007), no. 11, 2061-2068.

[14] Q. Yue, On tame kernel and class group in terms of quadratic forms, J. Number Theory 96 (2002), no. 2, 373-387. 
[15] _ 8-ranks of class groups of quadratic number fields and their densities, Acta Matematica Sinica (Eng. Ser.), to apppear.

[16] Q. Yue and J. Yu, The densities of 4-ranks of tame kernels for quadratic fields, J. Reine Angew. Math. 567 (2004), 151-173.

HWANYUP JUNG

Department of Mathematics Education

Chungbuk National University

Cheongue 361-763, Korea

E-mail address: hyjung@chungbuk.ac.kr

QIN YUE

Department of Mathematics

Nanjing University of Aeronautics and Astronautics

NANJing, 210016, P. R. China

E-mail address: yueqin@nuaa.edu.cn 